\title{
Bubbling solutions for an elliptic equation with exponential Neumann data in $\mathbb{R}^{2}$
}

\author{
Shengbing Deng And Monica Musso
}

Abstract. Let $\Omega$ be a bounded domain in $\mathbb{R}^{2}$ with smooth boundary; we study the following Neumann problem

$$
\begin{cases}-\Delta u+u=0 & \text { in } \Omega \\ \frac{\partial u}{\partial v}=\lambda u^{p-1} e^{u^{p}} & \text { on } \partial \Omega,\end{cases}
$$

where $v$ is the outer normal vector of $\partial \Omega, \lambda>0$ is a small parameter and $0<$ $p<2$. We construct bubbling solutions to problem (0.1) by a Lyapunov-Schmidt reduction procedure.

Mathematics Subject Classification (2010): 35B10 (primary); 35B33, 35J08, 58J05 (secondary).

\section{Introduction}

In this paper we consider the boundary-value problem

$$
\begin{cases}-\Delta u+u=0 & \text { in } \Omega \\ \frac{\partial u}{\partial v}=\lambda u^{p-1} e^{u^{p}} & \text { on } \partial \Omega,\end{cases}
$$

where $\Omega$ is a bounded domain in $\mathbb{R}^{2}$ with smooth boundary, $v$ is the outer normal vector of $\partial \Omega, \lambda>0$ is a small parameter, and $0<p<2$.

In [6] Dávila-del Pino-Musso analyzed the asymptotic behavior of solutions to problem (1.1) when $p=1$. Namely, they considered the following problem:

$$
\begin{cases}-\Delta u+u=0 & \text { in } \Omega \\ \frac{\partial u}{\partial v}=\lambda e^{u} & \text { on } \partial \Omega .\end{cases}
$$

The research of the second author has been partly supported by Fondecyt Grant 1120151 and CAPDE-Anillo ACT-125, Chile.

Received April 13,2012; accepted in revised form August 7, 2012. 
Suppose that $u_{\lambda}$ is a family of solutions to (1.2), with the property that $\lambda \int_{\partial \Omega} e^{u_{\lambda}}$ is bounded as $\lambda \rightarrow 0$; then there is an integer $k \geq 1$ such that, up to subsequences,

$$
\lim _{\lambda \rightarrow 0} \lambda \int_{\partial \Omega} e^{u_{\lambda}}=2 k \pi
$$

Moreover, there are $k$ distinct points $\xi_{j}$, with $j=1, \ldots, k$, on the boundary of $\Omega$, such that $\lambda e^{u_{\lambda}}$ approaches the sum of $k$ Dirac masses centered at these points $\xi_{j}$. The location of such points can be characterized as that of the critical points of the function given by

$$
\varphi_{k}\left(\xi_{1}, \ldots, \xi_{k}\right)=-\left[\sum_{j=1}^{k} H\left(\xi_{j}, \xi_{j}\right)+\sum_{l \neq j} G\left(\xi_{l}, \xi_{j}\right)\right],
$$

where $G(x, y)$ is the Green function of the problem

$$
\begin{cases}-\Delta_{x} G(x, y)+G(x, y)=0 & x \in \Omega \\ \frac{\partial G(x, y)}{\partial v_{x}}=2 \pi \delta_{y}(x) & x \in \partial \Omega,\end{cases}
$$

and $H$ is its regular part defined by

$$
H(x, y)=G(x, y)-2 \log \frac{1}{|x-y|} .
$$

The authors in [6] also proved the existence of solutions with the above properties. More precisely, they showed that, for any $k \geq 1$, Problem (1.2) has a solution $u_{\lambda}$, for all $\lambda$ small enough, with the property that

$$
\lambda \int_{\partial \Omega} e^{u_{\lambda}} d x \rightarrow 2 k \pi \quad \text { as } \quad \lambda \rightarrow 0 .
$$

These solutions develop $k$ peaks, as $\lambda \rightarrow 0$, around $k$ points of the boundary of $\Omega$, corresponding to the critical points of the function $\varphi_{k}$ defined in (1.4).

In this paper we will extend this result and we will consider the problem of the existence of solutions to (1.1) for any value of $p$ with $0<p<2$. In particular we will recover the result in [6], for $p=1$. Problem (1.1) is the Euler-Lagrange equation for the functional $J_{\lambda}: H^{1}(\Omega) \rightarrow \mathbb{R}$ defined by

$$
J_{\lambda}(u)=\frac{1}{2} \int_{\Omega}\left(|\nabla u|^{2}+u^{2}\right)-\frac{\lambda}{p} \int_{\partial \Omega} e^{u^{p}} .
$$

By the Young and Hölder inequalities, we know that $J_{\lambda}$ corresponds to the critical Trudinger-Moser trace embedding

$$
H^{1}(\Omega) \ni u \mapsto e^{u^{2}} \in L^{q}(\partial \Omega) \quad \forall q \geq 1,
$$


which is connected to the following critical Trudinger-Moser trace inequalities

$$
S_{\alpha}:=\sup \left\{\int_{\partial \Omega} e^{\alpha u^{2}}: u \in H^{1}(\Omega) \backslash\{0\},\|u\|_{H^{1}} \leq 1, \int_{\partial \Omega} u=0\right\}<\infty
$$

for any $\alpha \leq \pi$, see [1]. Multiplying by a suitable test function, we can find that smallness of $\lambda$ is necessary for the existence of a solution. From (1.7), there is a minimizer solution near zero. On the other hand, there is a second solution for (1.1) by the Mountain Pass Theorem.

Let $\varepsilon$ be the parameter, which depends on $\lambda$, defined by the relation

$$
p \lambda\left(-\frac{2}{p} \log \varepsilon\right)^{\frac{2(p-1)}{p}} \varepsilon^{\frac{p-2}{p}}=1 .
$$

Observe that, as $\lambda \rightarrow 0$, we have $\varepsilon \rightarrow 0$. Furthermore $\varepsilon=\lambda$ when $p=1$.

Our main result is the following:

Theorem 1.1. Assume $0<p<2$. For any integer $k \geq 1$ problem (1.1) has two families of solutions $u_{i, \lambda}$, for $i=1,2$ and for all $\lambda$ small enough, such that

$$
\lim _{\lambda \rightarrow 0} \varepsilon^{\frac{2-p}{p}} \int_{\partial \Omega} e^{u_{i, \lambda}^{p}}=2 k \pi
$$

where $\varepsilon$ is given by (1.8). Furthermore, there exist two families of points $\xi_{\lambda}^{i}=$ $\left(\xi_{1, \lambda}^{i}, \ldots, \xi_{k, \lambda}^{i}\right) \in(\partial \Omega)^{k}$, for $i=1,2$, with

$$
\left|\xi_{l, \lambda}^{i}-\xi_{j, \lambda}^{i}\right|>\delta \quad \text { for } \quad l \neq j \quad \text { and } \quad i=1,2
$$

for some small but fixed number $\delta>0$, such that $\xi_{\lambda}^{i} \rightarrow \xi^{i}$ as $\lambda \rightarrow 0$, with

$$
\nabla \varphi_{k}\left(\xi_{1}^{i}, \ldots, \xi_{k}^{i}\right)=0
$$

and

$$
u_{i, \lambda}(x)=p^{-\frac{1}{2}} \sqrt{\lambda} \varepsilon^{\frac{p-2}{2 p}}\left[\sum_{j=1}^{k} G\left(x, \xi_{j, \lambda}^{i}\right)+o(1)\right]
$$

where $o(1) \rightarrow 0$ on each compact subset of $\bar{\Omega} \backslash\left\{\xi_{1}^{i}, \ldots, \xi_{k}^{i}\right\}$. Moreover

$$
J_{\lambda}\left(u_{i, \lambda}\right)=\lambda \varepsilon^{\frac{p-2}{p}}\left[-\frac{2 k \pi}{p}+\frac{2 k \pi}{p} \log \frac{1}{\varepsilon}+\frac{\pi}{2-p} \varphi_{k}\left(\xi_{\lambda}^{i}\right)+|\log \varepsilon|^{-1} O(1)\right]
$$

where $O(1)$ is uniformly bounded as $\lambda \rightarrow 0$. 
The proof of our result relies on a very well known Lyapunov-Schmidt reduction procedure, introduced in $[2,15]$ and used in many different contexts, see for instance [5-14,17]. A key step in this procedure is to find a good first approximation for the solution. Usually, this ansatz is built as a sum of terms, each one of which turns out to solve an associate limit problem, properly scaled and translated. For our problem, the limit problem is

$$
\left\{\begin{array}{l}
\Delta v=0 \quad \text { in } \mathbb{R}_{+}^{2} \\
\frac{\partial v}{\partial v}=e^{v} \text { on } \partial \mathbb{R}_{+}^{2} \\
\int_{\partial \mathbb{R}_{+}^{2}} e^{v}<\infty .
\end{array}\right.
$$

A family of solutions to (1.12) is given by

$$
w_{t, \mu}(x)=w_{t, \mu}\left(x_{1}, x_{2}\right)=\log \frac{2 \mu}{\left(x_{1}-t\right)^{2}+\left(x_{2}+\mu\right)^{2}},
$$

where $t \in \mathbb{R}$ and $\mu>0$ are parameters. Set

$$
w_{\mu}(x):=w_{0, \mu}(x)=\log \frac{2 \mu}{x_{1}^{2}+\left(x_{2}+\mu\right)^{2}} .
$$

If we use the above solution, properly scaled, and centered at several points on the boundary of the domain, as our approximate solution, we get a very good approximation of a solution in a region far away from the points. In other words, the error is relatively small far away from these points. Close to the concentration points on the boundary, this approximation turns out to be good enough for our construction only when $p=1$. We refer to [6]. Unfortunately this is not the case when $p \in(0,2)$ and $p \neq 1$. In fact, in this case, we need to further improve the approximation near the concentration points. We do this by adding two other terms in the expansion of the solution: this can be done in a very natural way, which has first been used, for instance, in [12] for studying the problem

$$
\begin{cases}\Delta u+u^{q}=0, u>0 & \text { in } \Omega \\ u=0 & \text { on } \partial \Omega,\end{cases}
$$

where $\Omega$ is a smooth bounded domain in $\mathbb{R}^{2}$, and $q$ is a large exponent. Later on, this method has been applied in other contexts, see $[5,13,14,17]$. In particular, H. Castro in [5] used this method to study the Neumann problem

$$
\begin{cases}-\Delta u+u=0, u>0 & \text { in } \Omega \\ \frac{\partial u}{\partial v}=u^{q} & \text { on } \partial \Omega,\end{cases}
$$


where $\Omega$ is a bounded domain in $\mathbb{R}^{2}$ with smooth boundary $\partial \Omega$, $v$ is the outer normal vector to $\partial \Omega$, and $q$ is a large exponent. He showed that, if $q>1$ is a large parameter, for any integer $k \geq 1$, there exists at least two families of solutions $u_{q}$, which develop exactly $k$ peaks $\xi_{j} \in \partial \Omega$, and in the sense that $q u_{q}^{q} \rightarrow 2 e \pi \sum_{j=1}^{k} \delta_{\xi_{j}}$ as $q \rightarrow+\infty$.

It is important to remark about the analogy existing between our result and the Dirchlet problem

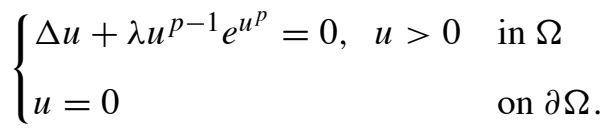

For $p=1$, the asymptotic behaviour of solutions to (1.17) for which $\lambda \int_{\Omega} e^{u}$ remains uniformly bounded as $\lambda \rightarrow 0$ is well understood after the works $[4,16,18]$ : indeed, $\lambda e^{u}$ approaches a superposition of Dirac deltas centered at points in the interior of $\Omega$. On the other hand, the construction of solutions with this behaviour has been achieved in $[3,8,11]$. In our previous paper [10] we constructed bubbling solutions to (1.17) for the whole range $0<p<2$. We showed that If $\Omega$ is not simply connected and if $k \geq 1$ is any integer, then for all small $\lambda$ Problem (1.17) has a solution concentrating around $k$ points in the interior of $\Omega$. In fact this result was shown first in [8] for $p=1$. Construction of bubbling solutions for Problem (1.17) and $p=2$ has been treated in [9]: in this case, the existence of such a solution is not only related to the location of the concentration points, but also to the rate of concentration at these points.

This paper is organized as follows: Section 2 is devoted to describing a first approximation solution to problem (1.1) and to estimating the error. Furthermore, problem (1.1) is rewritten as a fixed-point problem, involving a linear operator. In Section 3 we study the invertibility of the linear problem. In Section 4 we study the nonlinear problem. In Section 5 we study the variational reduction, and we prove the main Theorem 1.1 in Section 6. We will give some estimates in the Appendix, Section 7.

In this paper, the symbol $C$ denotes a generic positive constant independent of $\lambda$, that can change from one line to another. The symbols $O(t)$ (respectively $o(t)$ ) will denote quantities for which $\frac{O(t)}{|t|}$ stays bounded (respectively, $\frac{o(t)}{|t|}$ tends to zero) as the parameter $t$ goes to zero. In particular, we will often use the notation $o(1)$ for a quantity which tends to zero as $t \rightarrow 0$.

\section{Preliminaries and ansatz for the solution}

For any parameter $\varepsilon>0$, we can produce a solution to

$$
\begin{cases}\Delta u=0 & \text { in } \mathbb{R}_{+}^{2} \\ \frac{\partial u}{\partial v}=\varepsilon e^{u} & \text { on } \partial \mathbb{R}_{+}^{2},\end{cases}
$$


by taking

$$
u(x)=w_{\mu}(x / \varepsilon)-2 \log \varepsilon=\log \frac{2 \mu}{x_{1}^{2}+\left(x_{2}+\varepsilon \mu\right)^{2}},
$$

where $w_{\mu}$ is defined by (1.14). Based on this observation, we choose a sufficiently small but fixed number $\delta>0$ and assume to be given $k$ points $\xi_{j}, j=1, \ldots, k$, on $\partial \Omega$, satisfying

$$
\left|\xi_{l}-\xi_{j}\right|>\delta, \quad \text { for } l \neq j
$$

Furthermore, we consider $k$ positive numbers $\mu_{j}$ such that

$$
\delta<\mu_{j}<\delta^{-1}, \quad \text { for all } j=1, \ldots, k
$$

We define

$$
u_{j}(x)=\log \frac{2 \mu_{j}}{\left|x-\xi_{j}-\varepsilon \mu_{j} \nu\left(\xi_{j}\right)\right|^{2}} .
$$

We define the first approximate solution by

$$
U(x)=\frac{1}{p \gamma^{p-1}} \sum_{j=1}^{k}\left[u_{j}(x)+H_{j}^{\varepsilon}(x)\right],
$$

with some number $\gamma$, to be defined later on, where $H_{j}^{\varepsilon}$ is a correction term given as the solution of

$$
\begin{cases}-\Delta H_{j}^{\varepsilon}+H_{j}^{\varepsilon}=-u_{j} & \text { in } \Omega \\ \frac{\partial H_{j}^{\varepsilon}}{\partial v}=\varepsilon e^{u_{j}}-\frac{\partial u_{j}}{\partial v} & \text { on } \partial \Omega .\end{cases}
$$

We have the validity of the following lemma.

Lemma 2.1. Assume (2.2) and (2.3). For any $0<\alpha<1$, one has

$$
H_{j}^{\varepsilon}(x)=H\left(x, \xi_{j}\right)-\log \left(2 \mu_{j}\right)+O\left(\varepsilon^{\alpha}\right)
$$

uniformly in $\bar{\Omega}$, where $H$ is the regular part of Green's function defined (1.5).

We will give the proof of this lemma in the Appendix, Section 7.

We shall show later on that $U(x)$ is a good approximation for a solution to (1.1) far from the points $\xi_{j}$, but unfortunately, when $p \neq 1$, it is not good enough for our construction close to the points $\xi_{j}$. This is the reason why we need to further adjust this ansatz. In order to do this, let us first introduce the following result, whose proof is given in [6]. 
Proposition 2.2. Any bounded solution of the following problem

$$
\begin{cases}\Delta \phi=0 & \text { in } \mathbb{R}_{+}^{2} \\ \frac{\partial \phi}{\partial v}-e^{w_{\mu}} \phi=0 & \text { on } \partial \mathbb{R}_{+}^{2},\end{cases}
$$

is a linear combination of

$$
z_{0 \mu}(x)=-\frac{1}{\mu}\left(x \cdot \nabla w_{\mu}(x)+1\right)=\frac{1}{\mu}-2 \frac{x_{2}+\mu}{x_{1}^{2}+\left(x_{2}+\mu\right)^{2}},
$$

and

$$
z_{1 \mu}(x)=\frac{\partial w_{\mu}}{\partial x_{1}}=-2 \frac{x_{1}}{x_{1}^{2}+\left(x_{2}+\mu\right)^{2}} .
$$

Now, let us consider the following problem

$$
\begin{cases}\Delta \phi=0 & \text { in } \mathbb{R}_{+}^{2} \\ \frac{\partial \phi}{\partial v}-e^{w_{\mu}} \phi=e^{w_{\mu}} g & \text { on } \partial \mathbb{R}_{+}^{2},\end{cases}
$$

with $w_{\mu}$ defined in (1.14). In [5] it is showed that

Proposition 2.3. Let g be a $C^{1}\left(\partial \mathbb{R}_{+}^{2}\right)$ function such that, for $\mu>0, k \geq 0$, satisfies

$$
g(x)=O\left(\log ^{k}(1+|x|)\right) \quad \text { as }|x| \rightarrow \infty,
$$

and

$$
\int_{\partial \mathbb{R}_{+}^{2}} e^{w_{\mu}} g z_{0 \mu}=0=\int_{\partial \mathbb{R}_{+}^{2}} e^{w_{\mu}} g z_{1 \mu} .
$$

Then (2.9) has a solution $\phi \in C^{\alpha}\left(\mathbb{R}_{+}^{2}\right)$. Moreover, for any $0<\alpha<1$, and $|x| \rightarrow \infty$,

$$
|\phi(x)| \leq C \frac{1}{|x|^{\alpha}}, \quad|\nabla \phi(x)| \leq C \frac{1}{|x|^{1+\alpha}}, \quad\left|\nabla^{2} \phi(x)\right| \leq C \frac{1}{|x|^{2+\alpha}},
$$

where $C$ is a positive constant, which depends on $\|g\|_{L^{p}\left(\partial \mathbb{R}_{+}^{2}\right)}$, for some $p=$ $p(\alpha)>1$.

Let us define $\phi_{1 j}$ to be the solution of the problem

$$
\begin{cases}\Delta \phi_{1 j}=0 & \text { in } \mathbb{R}_{+}^{2} \\ \frac{\partial \phi_{1 j}}{\partial v}-e^{w_{\mu_{j}}} \phi_{1 j}=e^{w_{\mu_{j}}} g_{1} & \text { on } \partial \mathbb{R}_{+}^{2},\end{cases}
$$


where $w_{\mu_{j}}(y)=\log \frac{2 \mu_{j}}{y_{1}^{2}+\left(y_{2}+\mu_{j}\right)^{2}}$ and

$$
g_{1}=\alpha_{1 j}\left(w_{\mu_{j}}-1\right)+w_{\mu_{j}}+\frac{1}{2}\left(w_{\mu_{j}}\right)^{2}
$$

with $\alpha_{1 j}$ is a constant to be fixed, in terms of $\mu_{j}$. Observe first that, by definition, the function $g_{1}$ satisfies (2.10). We now choose $\alpha_{1 j}$ such that the orthogonality condition (2.11) holds. First we observe that $g_{1}$ is a symmetric function for any choice of $\alpha_{1 j}$, hence

$$
\int_{\partial \mathbb{R}_{+}^{2}} e^{w_{\mu_{j}}} g_{1} z_{1 \mu_{j}}=0
$$

Next, we chose the parameter $\alpha_{1 j}$ such that the other orthogonality condition is satisfied. Since

$$
\begin{aligned}
& \int_{\partial \mathbb{R}_{+}^{2}} e^{w_{\mu_{j}}} g_{1} z_{0 \mu_{j}} \\
& =-\frac{1}{\mu_{j}} \int_{\partial \mathbb{R}_{+}^{2}} e^{w_{\mu_{j}}}\left(\alpha_{1 j}\left(w_{\mu_{j}}-1\right)+w_{\mu_{j}}+\frac{1}{2}\left(w_{\mu_{j}}\right)^{2}\right)\left(y \cdot \nabla w_{\mu_{j}}(y)+1\right) \\
& =-\frac{1}{\mu_{j}} \alpha_{1 j} \int_{-\infty}^{\infty}\left(e^{w_{\mu_{j}}\left(y_{1}, 0\right)}\left(w_{\mu_{j}}\left(y_{1}, 0\right)-1\right) \frac{\partial w_{\mu_{j}}}{\partial y_{1}}\left(y_{1}, 0\right) y_{1}\right. \\
& \left.\quad+e^{w_{\mu_{j}}\left(y_{1}, 0\right)}\left(w_{\mu_{j}}\left(y_{1}, 0\right)-1\right)\right) d y_{1} \\
& -\frac{1}{\mu_{j}} \int_{-\infty}^{\infty}\left(e^{w_{\mu_{j}}\left(y_{1}, 0\right)} w_{\mu_{j}}\left(y_{1}, 0\right) \frac{\partial w_{\mu_{j}}}{\partial y_{1}}\left(y_{1}, 0\right) y_{1}+e^{w_{\mu_{j}}\left(y_{1}, 0\right)} w_{\mu_{j}}\left(y_{1}, 0\right)\right) d y_{1} \\
& -\frac{1}{\mu_{j}} \int_{-\infty}^{\infty}\left(e^{w_{\mu_{j}}\left(y_{1}, 0\right)} \frac{\left(w_{\mu_{j}}\right)^{2}}{2}\left(y_{1}, 0\right) \frac{\partial w_{\mu_{j}}}{\partial y_{1}}\left(y_{1}, 0\right) y_{1}+e^{w_{\mu_{j}}\left(y_{1}, 0\right)} \frac{\left(w_{\mu_{j}}\right)^{2}}{2}\left(y_{1}, 0\right)\right) d y_{1} \\
& =-\frac{1}{\mu_{j}}\left[\alpha_{1 j} \int_{-\infty}^{\infty} e^{w_{\mu_{j}}\left(y_{1}, 0\right)} d y_{1}+\int_{-\infty}^{\infty} e^{w_{\mu_{j}}\left(y_{1}, 0\right)} w_{\mu_{j}}\left(y_{1}, 0\right) d y_{1}\right] .
\end{aligned}
$$

Thus we define $\alpha_{1 j}$ such that

$$
\alpha_{1 j} \int_{-\infty}^{\infty} e^{w_{\mu_{j}}\left(y_{1}, 0\right)} d y_{1}+\int_{-\infty}^{\infty} e^{w_{\mu_{j}}\left(y_{1}, 0\right)} w_{\mu_{j}}\left(y_{1}, 0\right) d y_{1}=0
$$

Since

$$
\int_{-\infty}^{\infty} e^{w_{\mu_{j}}\left(y_{1}, 0\right)} d y_{1}=\int_{-\infty}^{\infty} \frac{2 \mu_{j}}{y_{1}^{2}+\mu_{j}^{2}} d y_{1}=2 \int_{-\infty}^{\infty} \frac{1}{t^{2}+1} d t=2 \pi
$$


and

$$
\begin{aligned}
& \int_{-\infty}^{\infty} e^{w_{\mu_{j}}\left(y_{1}, 0\right)} w_{\mu_{j}}\left(y_{1}, 0\right) d y_{1} \\
& =\int_{-\infty}^{\infty} \frac{2 \mu_{j}}{y_{1}^{2}+\mu_{j}^{2}} \log \frac{2 \mu_{j}}{y_{1}^{2}+\mu_{j}^{2}} d y_{1} \\
& =2 \int_{-\infty}^{\infty} \frac{1}{t^{2}+1}\left[\log \frac{1}{t^{2}+1}+\log \left(2 \mu_{j}^{-1}\right)\right] d t=-2 \pi \log \left(2 \mu_{j}\right) .
\end{aligned}
$$

Here we use the following fact (see the proof in the Appendix)

$$
\int_{-\infty}^{\infty} \frac{1}{t^{2}+1} \log \frac{1}{t^{2}+1} d t=-2 \pi \log 2 .
$$

Therefore, $\alpha_{1 j}$ is given by

$$
\alpha_{1 j}=\log \left(2 \mu_{j}\right) \text {. }
$$

Then we get the existence of $\phi_{1 j}$ by Proposition 2.3. With this function, we define

$$
w_{1 j}(y)=\phi_{1 j}(y)+\alpha_{1 j} w_{\mu_{j}}(y) .
$$

We observe that $w_{1 j}$ satisfies

$$
\begin{cases}\Delta w_{1 j}=0 & \text { in } \mathbb{R}_{+}^{2} \\ \frac{\partial w_{1 j}}{\partial v}-e^{w_{\mu_{j}}} w_{1 j}=e^{w_{\mu_{j}}}\left(w_{\mu_{j}}+\frac{1}{2}\left(w_{\mu_{j}}\right)^{2}\right) & \text { on } \partial \mathbb{R}_{+}^{2}\end{cases}
$$

Next, assume for the moment that $p \neq 1$ and consider $\phi_{2 j}$, a solution of

$$
\begin{cases}\Delta \phi_{2 j}=0 & \text { in } \mathbb{R}_{+}^{2} \\ \frac{\partial \phi_{2 j}}{\partial v}-e^{w_{\mu_{j}}} \phi_{2 j}=e^{w_{\mu_{j}} g_{2}} & \text { on } \partial \mathbb{R}_{+}^{2}\end{cases}
$$

where

$$
\begin{aligned}
g_{2}= & \alpha_{2 j}\left(w_{\mu_{j}}-1\right)+w_{1 j}+\frac{p-2}{2(p-1)}\left(w_{\mu_{j}}\right)^{2}+\frac{1}{2}\left(w_{1 j}\right)^{2} \\
& +\frac{1}{8}\left(w_{\mu_{j}}\right)^{4}+w_{\mu_{j}} w_{1 j}+\frac{1}{2}\left(w_{\mu_{j}}\right)^{3}+\frac{1}{2} w_{1 j}\left(w_{\mu_{j}}\right)^{2} .
\end{aligned}
$$

Again, by definition, we observe that $g_{2}$ satisfies (2.10) and it is a symmetric function. Thus, arguing as before, we can find a constant $\alpha_{2 j}$ such that the orthogonality conditions (2.11) are satisfied. Then we have the existence of function $\phi_{2 j}$ by Proposition 2.3. 
For $\xi_{j} \in \partial \Omega$, let $\delta>0$ be a fixed small radius, depending only in the geometry of $\Omega$, such that

$$
F_{j}: B_{\delta}(0) \cap\left(\Omega-\xi_{j}\right) \rightarrow M \cap \mathbb{R}_{+}^{2},
$$

is a $C^{2}$ diffeomorphism, and $M$ an open neighborhood of the origin such that

$$
F_{j}\left(B_{\delta}(0) \cap\left(\partial \Omega-\xi_{j}\right)\right) \subseteq M \cap \partial \mathbb{R}_{+}^{2} .
$$

We can select $F_{j}$ so that it preserves the area. For $i=1,2$, define

$$
\begin{aligned}
\tilde{w}_{i j}(x) & =\phi_{i j}\left(\frac{F_{j}\left(x-\xi_{j}\right)}{\varepsilon}\right)+\alpha_{i j} w_{\mu_{j}}\left(\frac{x-\xi_{j}}{\varepsilon}\right) \\
& :=\tilde{\phi}_{i j}(y)+\alpha_{i j} \tilde{w}_{j}(y),
\end{aligned}
$$

where

$$
\tilde{w}_{j}(y):=w_{\mu_{j}}\left(y-\xi_{j}^{\prime}\right)=\log \frac{2 \mu_{j}}{\left|y-\xi_{j}^{\prime}-\mu_{j} \nu\left(\xi_{j}^{\prime}\right)\right|^{2}},
$$

with $\xi_{j}^{\prime}=\xi_{j} / \varepsilon$ and where we will write $v$ for the exterior normal unit vector to $\partial \Omega$ and $\partial \Omega_{\varepsilon}$. Here $\Omega_{\varepsilon}=\varepsilon^{-1} \Omega$. Then, for any $0<p<2$, let us define the first approximation solution to (1.1) is

$$
\begin{aligned}
U_{\lambda}(x)= & \frac{1}{p \gamma^{p-1}} \sum_{j=1}^{k}\left[u_{j}(x)+H_{j}^{\varepsilon}(x)+\frac{p-1}{p} \frac{1}{\gamma^{p}}\left(\tilde{w}_{1 j}(x)+H_{1 j}^{\varepsilon}(x)\right)\right. \\
& \left.+\left(\frac{p-1}{p}\right)^{2} \frac{1}{\gamma^{2 p}}\left(\tilde{w}_{2 j}(x)+H_{2 j}^{\varepsilon}(x)\right)\right]
\end{aligned}
$$

where $H_{i j}^{\varepsilon}, i=1,2$, is a new correction term, given by the solution of

$$
\begin{cases}-\Delta H_{i j}^{\varepsilon}+H_{i j}^{\varepsilon}=-\alpha_{i j} \tilde{w}_{j}(x / \varepsilon) & \text { in } \Omega \\ \frac{\partial H_{i j}^{\varepsilon}}{\partial v}=\alpha_{i j}\left(\varepsilon e^{u_{j}}-\frac{\partial u_{j}}{\partial v}\right) & \text { on } \partial \Omega .\end{cases}
$$

By the same arguments used in Lemma 2.1, we have the following result, whose proof is postponed to the Appendix, Section 7.

Lemma 2.4. For any $0<\alpha<1$, for $i=1,2$, one has

$$
H_{i j}^{\varepsilon}(x)=\alpha_{i j} H\left(x, \xi_{j}\right)-\alpha_{i j} \log \left(2 \mu_{j}\right)-2 \alpha_{i j} \log \varepsilon+O\left(\varepsilon^{\alpha}\right)
$$

uniformly in $\bar{\Omega}$, where $H$ is the regular part of Green's function defined (1.5). 
Consider now the change of variables

$$
v(y)=p \gamma^{p-1} u(\varepsilon y)-p \gamma^{p}, \quad \text { with } \gamma^{p}=-\frac{2}{p} \log \varepsilon .
$$

Then under the choice of $\varepsilon$ in (1.8), problem (1.1) reduces to

$$
\begin{cases}-\Delta v+\varepsilon^{2} v=2 \varepsilon^{2} \log \varepsilon & \text { in } \Omega_{\varepsilon} \\ \frac{\partial v}{\partial v}=f(v) & \text { on } \partial \Omega_{\varepsilon},\end{cases}
$$

where $\Omega_{\varepsilon}=\varepsilon^{-1} \Omega$, and

$$
f(v)=\left(1+\frac{v}{p \gamma^{p}}\right)^{p-1} e^{\gamma^{p}\left[\left(1+\frac{v}{p \gamma^{p}}\right)^{p}-1\right]} .
$$

Let us define the first approximation solution to (2.21) as

$$
V_{\lambda}(y)=p \gamma^{p-1} U_{\lambda}(\varepsilon y)-p \gamma^{p}
$$

with $U_{\lambda}$ defined by (2.18).

We next describe $V_{\lambda}$ close to the points $\xi_{j}^{\prime}$. We write $y=\varepsilon^{-1} x, \xi_{j}^{\prime}=\varepsilon^{-1} \xi_{j}$. For $\left|x-\xi_{j}\right|<\delta$ with $\delta$ sufficiently small but fixed, by Lemmas 2.1 and 2.4 , and the fact $u_{j}(\varepsilon y)-p \gamma^{p}=\tilde{w}_{j}(y)$, we have

$$
\begin{aligned}
V_{\lambda}(y) & u_{j}(\varepsilon y)+H_{j}^{\varepsilon}(\varepsilon y)+\frac{p-1}{p} \frac{1}{\gamma^{p}}\left(\tilde{w}_{1 j}(\varepsilon y)+H_{1 j}^{\varepsilon}(\varepsilon y)\right) \\
& +\left(\frac{p-1}{p}\right)^{2} \frac{1}{\gamma^{2 p}}\left(\tilde{w}_{2 j}(\varepsilon y)+H_{2 j}^{\varepsilon}(\varepsilon y)\right)-p \gamma^{p} \\
& +\sum_{l \neq j}^{k}\left(\tilde{w}_{l}(\varepsilon y)+H_{l}^{\varepsilon}(\varepsilon y)+\frac{p-1}{p} \frac{1}{\gamma^{p}}\left(\tilde{w}_{1 l}(\varepsilon y)+H_{1 l}^{\varepsilon}(\varepsilon y)\right)\right. \\
& \left.+\left(\frac{p-1}{p}\right)^{2} \frac{1}{\gamma^{2 p}}\left(\tilde{w}_{2 l}(\varepsilon y)+H_{2 l}^{\varepsilon}(\varepsilon y)\right)\right) \\
= & \tilde{w}_{j}(y)+\frac{p-1}{p} \frac{1}{\gamma^{p}} \tilde{w}_{1 j}(\varepsilon y)+\left(\frac{p-1}{p}\right)^{2} \frac{1}{\gamma^{2 p}} \tilde{w}_{2 j}(\varepsilon y)+O\left(\varepsilon\left|y-\xi_{j}^{\prime}\right|\right)+O\left(\varepsilon^{\alpha}\right) \\
& -\log \left(2 \mu_{j}\right)+\left[1+\alpha_{1 j} \frac{p-1}{p} \frac{1}{\gamma^{p}}+\alpha_{2 j}\left(\frac{p-1}{p}\right)^{2} \frac{1}{\gamma^{2 p}}\right] \\
& \left.\times\left(H\left(\xi_{j}, \xi_{j}\right)+\sum_{l \neq j}^{k} G_{(\xi l}, \xi_{j}\right)\right) \\
& -\left[\alpha_{1 j} \frac{p-1}{p} \frac{1}{\gamma^{p}}+\alpha_{2 j}\left(\frac{p-1}{p}\right)^{2} \frac{1}{\gamma^{2 p}}\right]\left(\log \left(2 \mu_{j}\right)+2 \log \varepsilon\right) \\
& {[}
\end{aligned}
$$


We now choose the parameters $\mu_{j}$ : we assume they are defined by the relation

$$
\begin{aligned}
\log \left(2 \mu_{j}\right)= & \left(H\left(\xi_{j}, \xi_{j}\right)+\sum_{l \neq j}^{k} G\left(\xi_{l}, \xi_{j}\right)\right)+(p-1) \alpha_{1 j} \\
+ & \alpha_{1 j} \frac{p-1}{p} \frac{1}{\gamma^{p}}\left(H\left(\xi_{j}, \xi_{j}\right)+\sum_{l \neq j}^{k} G\left(\xi_{l}, \xi_{j}\right)\right. \\
& \left.-\log \left(2 \mu_{j}\right)+(p-1) \frac{\alpha_{2 j}}{\alpha_{1 j}}\right) \\
& +\alpha_{2 j}\left(\frac{p-1}{p}\right)^{2} \frac{1}{\gamma^{2 p}}\left(H\left(\xi_{j}, \xi_{j}\right)+\sum_{l \neq j}^{k} G\left(\xi_{l}, \xi_{j}\right)-\log \left(2 \mu_{j}\right)\right) .
\end{aligned}
$$

Taking into account the explicit expression (2.15) of the constant $\alpha_{1 j}$, we observe that $\mu_{j}$ bifurcates, as $\lambda$ goes to zero, from the value

$$
\bar{\mu}_{j}=\frac{1}{2} e^{\frac{1}{2-p}\left[H\left(\xi_{j}, \xi_{j}\right)+\sum_{l \neq j}^{k} G\left(\xi_{l}, \xi_{j}\right)\right]}
$$

solution of equation

$$
\log \left(2 \mu_{j}\right)=\left(H\left(\xi_{j}, \xi_{j}\right)+\sum_{l \neq j}^{k} G\left(\xi_{l}, \xi_{j}\right)\right)+(p-1) \alpha_{1 j}
$$

Thus, $\mu_{j}$ is a perturbation of order $\frac{1}{\gamma^{p}}$ of the value $\bar{\mu}_{j}$, namely

$$
\log \left(2 \mu_{j}\right)=\frac{1}{2-p}\left(H\left(\xi_{j}, \xi_{j}\right)+\sum_{l \neq j}^{k} G\left(\xi_{l}, \xi_{j}\right)\right)\left(1+O\left(\frac{1}{\gamma^{p}}\right)\right) .
$$

Then, by this choice of the parameters $\mu_{j}$, we deduce that, if $\left|y-\xi_{j}^{\prime}\right|<\delta / \varepsilon$ with $\delta$ sufficiently small but fixed, we can rewrite

$$
V_{\lambda}(y)=\tilde{w}_{j}(y)+\frac{p-1}{p} \frac{1}{\gamma^{p}} \tilde{w}_{1 j}(\varepsilon y)+\left(\frac{p-1}{p}\right)^{2} \frac{1}{\gamma^{2 p}} \tilde{w}_{2 j}(\varepsilon y)+\theta(y),
$$


with

$$
\theta(y)=O\left(\varepsilon\left|y-\xi_{j}^{\prime}\right|\right)+O\left(\varepsilon^{\alpha}\right) .
$$

We will look for solutions to (2.21) of the form

$$
v=V_{\lambda}+\phi
$$

where $V_{\lambda}$ is defined as in (2.23), and $\phi$ represents a lower order correction. We aim at finding a solution for small $\phi$ provided that the points $\xi_{j}$ are suitably chosen. For small $\phi$, we can rewrite problem (2.21) as a nonlinear perturbation of its linearization, namely,

$$
\begin{cases}-\Delta \phi+\varepsilon^{2} \phi=0 & x \in \Omega_{\varepsilon} \\ L(\phi)=E_{\lambda}+N(\phi) & x \in \partial \Omega_{\varepsilon},\end{cases}
$$

where

$$
\begin{aligned}
L(\phi) & :=\frac{\partial \phi}{\partial v}-f^{\prime}\left(V_{\lambda}\right) \phi, \\
E_{\lambda} & :=f\left(V_{\lambda}\right)-\frac{\partial V_{\lambda}}{\partial v}, \\
N(\phi) & :=f\left(V_{\lambda}+\phi\right)-f\left(V_{\lambda}\right)-f^{\prime}\left(V_{\lambda}\right) \phi .
\end{aligned}
$$

We recall that $f(t)=\left(1+\frac{t}{p \gamma^{p}}\right)^{p-1} e^{\gamma^{p}\left[\left(1+\frac{t}{p \gamma^{p}}\right)^{p}-1\right]}$.

In order to solve the problem (2.30), first we have to study the invertibility properties of the linear operator $L$. In order to do this, we introduce a weighted $L^{\infty}$-norm defined as

$$
\|h\|_{*, \partial \Omega_{\varepsilon}}:=\sup _{y \in \partial \Omega_{\varepsilon}}\left(\sum_{j=1}^{k}\left(1+\left|y-\xi_{j}^{\prime}\right|\right)^{-1-\sigma}+\varepsilon\right)^{-1}|h(y)|
$$

for any $h \in L^{\infty}\left(\partial \Omega_{\varepsilon}\right)$, where we fix $0<\sigma<1$ later on. With respect to this norm, the error term $E_{\lambda}$ given in (2.32) can be estimated in the following way:

Lemma 2.5. Let $\delta>0$ be a small but fixed number, assume (2.2) and (2.3). Then there exists $C>0$ such that

$$
\left\|E_{\lambda}\right\|_{*, \partial \Omega_{\varepsilon}} \leq \frac{C}{\gamma^{3 p}}=\frac{C}{|\log \varepsilon|^{3}}
$$

for all $\lambda$ small enough. 
Proof. Far away from the points $\xi_{j}$, namely for $\left|x-\xi_{j}\right|>\delta$, i.e. $\left|y-\xi_{j}^{\prime}\right|>\frac{\delta}{\varepsilon}$, for all $j=1, \ldots, k$, we have that

$$
\frac{\partial V_{\lambda}}{\partial v}=p \gamma^{p-1} \varepsilon \frac{\partial U_{\lambda}(\varepsilon y)}{\partial v}=O\left(\gamma^{p-1} \varepsilon^{2}\right)
$$

On the other hand, in this region we have

$$
1+\frac{V_{\lambda}(y)}{p \gamma^{p}}=1+\frac{2 \log \varepsilon+O(1)}{p \gamma^{p}}=\frac{O(1)}{|\log \varepsilon|}
$$

where $O(1)$ denotes a smooth function, uniformly bounded, as $\varepsilon \rightarrow 0$, in the considered region. Hence

$$
f\left(V_{\lambda}\right)=\left(1+\frac{V_{\lambda}}{p \gamma^{p}}\right)^{p-1} e^{\gamma^{p}\left[\left(1+\frac{V_{\lambda}}{p \gamma^{p}}\right)^{p}-1\right]}=\frac{\varepsilon^{\frac{2}{p}}}{\mid \log \varepsilon^{p-1}} O(1) .
$$

Hence if we are far away from the points $\xi_{j}$, or equivalently for $\left|y-\xi_{j}^{\prime}\right|>\frac{\delta}{\varepsilon}$, the size of the error, measured with respect to the $\|\cdot\|_{*, \partial \Omega_{\varepsilon}}$-norm, is relatively small. Namely, if we denote by $1_{\text {outer }}$ the characteristic function of the set $\left\{y:\left|y-\xi_{j}^{\prime}\right|>\right.$ $\left.\frac{\delta}{\varepsilon}, j=1, \ldots, k\right\}$, then in this region we have

$$
\left\|E_{\lambda} 1_{\text {outer }}\right\|_{*, \partial \Omega_{\varepsilon}} \leq C \frac{\varepsilon^{\frac{2-p}{p}}}{|\log \varepsilon|^{p-1}} .
$$

Let us now fix the index $j$ in $\{1, \ldots, k\}$, for $\left|y-\xi_{j}^{\prime}\right|<\frac{\delta}{\varepsilon}$, we have

$$
\begin{aligned}
\frac{\partial V_{\lambda}}{\partial v}= & e^{\tilde{w}_{j}(y)} \\
& +\frac{p-1}{p} \frac{1}{\gamma^{p}} \frac{\partial \tilde{w}_{1 j}(x)}{\partial v}+\left(\frac{p-1}{p}\right)^{2} \frac{1}{\gamma^{2 p}} \frac{\partial \tilde{w}_{2 j}(x)}{\partial v}+O\left(\varepsilon^{2}\right) \\
= & e^{\tilde{w}_{j}(y)}+\frac{p-1}{p} \frac{1}{\gamma^{p}}\left(\frac{\partial \tilde{\phi}_{1 j}(y)}{\partial v}+\alpha_{1 j} e^{\tilde{w}_{j}}\right) \\
& +\left(\frac{p-1}{p}\right)^{2} \frac{1}{\gamma^{2 p}}\left(\frac{\partial \tilde{\phi}_{2 j}(y)}{\partial v}+\alpha_{2 j} e^{\tilde{w}_{j}}\right)+O\left(\varepsilon^{2}\right) .
\end{aligned}
$$


On the other hand, for any $R>0$ large but fixed, in the ball $\left|y-\xi_{j}^{\prime}\right|<R_{\varepsilon}:=$ $R|\log \varepsilon|^{\alpha}$, with $\alpha \geq 3$, we can use the Taylor expansion to obtain

$$
\begin{aligned}
& f\left(V_{\lambda}\right) \\
& =\left(1+\frac{1}{p \gamma^{p}}\left(\tilde{w}_{j}(y)+\frac{p-1}{p} \frac{1}{\gamma^{p}} \tilde{w}_{1 j}(\varepsilon y)+\left(\frac{p-1}{p}\right)^{2} \frac{1}{\gamma^{2 p}} \tilde{w}_{2 j}(\varepsilon y)+\theta(y)\right)\right)^{p-1} \\
& \times e^{\gamma^{p}\left[\left(1+\frac{1}{p \gamma^{p}}\left(\tilde{w}_{j}(y)+\frac{p-1}{p} \frac{1}{\gamma^{p}} \tilde{w}_{1 j}(\varepsilon y)+\left(\frac{p-1}{p}\right)^{2} \frac{1}{\gamma^{2 p}} \tilde{w}_{2 j}(\varepsilon y)+\theta(y)\right)\right)^{p}-1\right]} \\
& =\left(1+\frac{p-1}{p} \frac{1}{\gamma^{p}} \tilde{w}_{j}(y)+\left(\frac{p-1}{p}\right)^{2} \frac{1}{\gamma^{2 p}} \tilde{w}_{1 j}(\varepsilon y)\right. \\
& \left.+\left(\frac{p-1}{p}\right)^{3} \frac{1}{\gamma^{3 p}} \tilde{w}_{2 j}(\varepsilon y)+\frac{p-1}{p} \frac{1}{\gamma^{p}} \theta(y)\right) \\
& \times e^{\tilde{w}_{j}(y)} e^{\frac{p-1}{p} \frac{1}{\gamma^{p}} \tilde{w}_{1 j}(\varepsilon y)} e^{\left(\frac{p-1}{p}\right)^{2} \frac{1}{\gamma^{2 p}} \tilde{w}_{2 j}(\varepsilon y)} e^{\theta(y)} \\
& \times e^{\frac{1}{2} \frac{p-1}{p} \frac{1}{\gamma^{p}}\left[\tilde{w}_{j}(y)+\frac{p-1}{p} \frac{1}{\gamma^{p}} \tilde{w}_{1 j}(\varepsilon y)+\left(\frac{p-1}{p}\right)^{2} \frac{1}{\gamma^{2 p}} \tilde{w}_{2 j}(\varepsilon y)+\theta(y)\right]^{2}} \\
& =e^{\tilde{w}_{j}(y)}+\frac{p-1}{p} \frac{1}{\gamma^{p}}\left\{e^{\tilde{w}_{j}} \tilde{w}_{1 j}(\varepsilon y)+e^{\tilde{w}_{j}}\left[\tilde{w}_{j}+\frac{1}{2}\left(\tilde{w}_{j}\right)^{2}\right]\right\}+\left(\frac{p-1}{p}\right)^{2} \frac{1}{\gamma^{2 p}} \\
& \times\left\{e^{\tilde{w}_{j}} \tilde{w}_{2 j}(\varepsilon y)+e^{\tilde{w}_{j}}\left[\tilde{w}_{1 j}+\frac{p-2}{2(p-1)}\left(\tilde{w}_{j}\right)^{2}+\frac{1}{2}\left(\tilde{w}_{1 j}\right)^{2}+\frac{1}{8}\left(\tilde{w}_{j}\right)^{4}\right.\right. \\
& \left.\left.+\tilde{w}_{j} \tilde{w}_{1 j}+\frac{1}{2}\left(\tilde{w}_{j}\right)^{3}+\frac{1}{2} \tilde{w}_{1 j}\left(\tilde{w}_{j}\right)^{2}\right]\right\} \\
& +\frac{p-1}{p} \frac{1}{\gamma^{p}} e^{\tilde{w}_{j}} \theta(y)+\left(\frac{p-1}{p}\right)^{2} \frac{1}{\gamma^{2 p}} e^{\tilde{w}_{j}}\left[\tilde{w}_{j}+\tilde{w}_{1 j}(\varepsilon y)\right] \theta(y) \\
& +\left(\frac{p-1}{p}\right)^{3} \frac{1}{\gamma^{3 p}}\left\{e^{\tilde{w}_{j}} \tilde{w}_{2 j}(\varepsilon y)+e^{\tilde{w}_{j}}\left[\frac{1}{6}\left(\tilde{w}_{1 j}\right)^{3}(\varepsilon y)+\frac{1}{48}\left(\tilde{w}_{j}\right)^{6}+\tilde{w}_{j} \tilde{w}_{1 j}(\varepsilon y)\right.\right. \\
& +\frac{1}{2}\left(\tilde{w}_{1 j}\right)^{2}(\varepsilon y)+2 \tilde{w}_{j} \tilde{w}_{2 j}(\varepsilon y)+\frac{1}{2} \tilde{w}_{1 j}(\varepsilon y)\left(\tilde{w}_{j}\right)^{3} \\
& +\frac{2 p-3}{2(p-1)} \tilde{w}_{j}\left(\tilde{w}_{1 j}\right)^{2}(\varepsilon y)+\frac{1}{8}\left(\tilde{w}_{j}\right)^{5}+\left(\tilde{w}_{1 j}\right)^{2}(\varepsilon y) \\
& +\frac{1}{2} \tilde{w}_{1 j}(\varepsilon y)\left(\tilde{w}_{j}\right)^{2}+\frac{p-2}{4(p-1)}\left(\tilde{w}_{j}\right)^{4}+\tilde{w}_{1 j}(\varepsilon y) \tilde{w}_{2 j}(\varepsilon y) \\
& \left.\left.+\frac{1}{8} \tilde{w}_{1 j}(\varepsilon y)\left(\tilde{w}_{j}\right)^{4}+\frac{1}{4}\left(\tilde{w}_{j} \tilde{w}_{1 j}(\varepsilon y)\right)^{2}+\frac{1}{2} \tilde{w}_{2 j}(\varepsilon y)\left(\tilde{w}_{j}\right)^{2}\right]\right\} \\
& +O\left(\frac{\log \left|y-\xi_{j}^{\prime}\right|}{\gamma^{3 p}}\right) \text {. }
\end{aligned}
$$


Thus, thanks to the fact that we have improved our original approximation with the terms $\tilde{w}_{1 j}(\varepsilon y)$ and $\tilde{w}_{2 j}(\varepsilon y)$, and the definition of $*$-norm, we get

$$
\left\|E_{\lambda} 1_{B\left(\xi_{j}^{\prime}, R_{\varepsilon}\right)}\right\|_{*, \partial \Omega_{\varepsilon}} \leq \frac{C}{\gamma^{3 p}}=\frac{C}{|\log \varepsilon|^{3}} .
$$

Here $1_{B\left(\xi_{j}^{\prime}, R_{\varepsilon}\right)}$ denotes the characteristic function of $B\left(\xi_{j}^{\prime}, R_{\varepsilon}\right)$. Finally, in the remaining region, namely where $R_{\varepsilon}<\left|y-\xi_{j}^{\prime}\right|<\frac{\delta}{\varepsilon}$, for any $j=1, \ldots, k$, we have from one hand that $\left|\frac{\partial V_{\lambda}(y)}{\partial v}\right| \leq C e^{\tilde{w}_{j}(y)}$, and also $\left|f\left(V_{\lambda}(y)\right)\right| \leq C e^{\tilde{w}_{j}(y)}$ as consequence of (2.24). These facts, together with (2.36) and (2.37) give estimate (2.35).

In the next two lemmas we prove some estimates that will be of use later on in our construction.

Lemma 2.6. Let $\delta>0$ be a small but fixed number, assume (2.2) and (2.3). Then there exists $C>0$ such that, for all small $\lambda$,

$$
\left\|f^{\prime}\left(V_{\lambda}\right)-e^{\tilde{w}_{j}}\right\|_{*, \partial \Omega_{\varepsilon}} \leq \frac{C}{|\log \varepsilon|}
$$

and there exists some positive constant $D_{0}$ such that

$$
f^{\prime}\left(V_{\lambda}\right) \leq D_{0} \sum_{j=1}^{k} e^{\tilde{w}_{j}}
$$

Proof. We have

$$
\begin{aligned}
f^{\prime}\left(V_{\lambda}\right)= & \frac{p-1}{p} \frac{1}{\gamma^{p}}\left(1+\frac{V_{\lambda}}{p \gamma^{p}}\right)^{p-2} e^{\gamma^{p}\left[\left(1+\frac{V_{\lambda}}{p \gamma^{p}}\right)^{p}-1\right]} \\
& +\left(1+\frac{V_{\lambda}}{p \gamma^{p}}\right)^{2(p-1)} e^{\gamma^{p}\left[\left(1+\frac{V_{\lambda}}{p \gamma^{p}}\right)^{p}-1\right]}:=I_{a}+I_{b}
\end{aligned}
$$

As in the proof of Lemma 2.5, far away from the points $\xi_{j}$, namely for $\left|x-\xi_{j}\right|>\delta$, i.e. $\left|y-\xi_{j}^{\prime}\right|>\frac{\delta}{\varepsilon}$, for all $j=1, \ldots, k$, we have

$$
1+\frac{V_{\lambda}(y)}{p \gamma^{p}}=1+\frac{2 \log \varepsilon+O(1)}{p \gamma^{p}}=\frac{O(1)}{|\log \varepsilon|} .
$$

Therefore

$$
I_{a}=\frac{\varepsilon^{\frac{2}{p}}}{|\log \varepsilon|^{p-1}} O(1), \quad \text { and } I_{b}=\frac{\varepsilon^{\frac{2}{p}}}{|\log \varepsilon|^{2(p-1)}} O(1)
$$


Then we have

$$
f^{\prime}\left(V_{\lambda}\right) 1_{\text {outer }}=\frac{\varepsilon^{\frac{2}{p}}}{|\log \varepsilon|^{p-1}} O(1) .
$$

On the other hand, fix the index $j$ in $\{1, \ldots, k\}$, for $\left|y-\xi_{j}^{\prime}\right|<R_{\varepsilon}$ with $R_{\varepsilon}=$ $R|\log \varepsilon|$, for any $R>0$ large but fixed; we use the Taylor expansion to get

$$
\begin{aligned}
& I_{a}=\frac{p-1}{p} \frac{1}{\gamma^{p}}\left(1+\frac{1}{p \gamma^{p}}\left(\tilde{w}_{j}(y)+\frac{p-1}{p} \frac{1}{\gamma^{p}} \tilde{w}_{1 j}(y)\right.\right. \\
& \left.\left.+\left(\frac{p-1}{p}\right)^{2} \frac{1}{\gamma^{2 p}} \tilde{w}_{2 j}(y)+\theta(y)\right)\right)^{p-2} \\
& \times e^{\gamma^{p}\left[\left(1+\frac{1}{p \gamma^{p}}\left(\tilde{w}_{j}(y)+\frac{p-1}{p} \frac{1}{\gamma^{p}} \tilde{w}_{1 j}(y)+\left(\frac{p-1}{p}\right)^{2} \frac{1}{\gamma^{2 p}} \tilde{w}_{2 j}(y)+\theta(y)\right)\right)^{p}-1\right]} \\
& =\frac{p-2}{p} \frac{1}{\gamma^{p}}\left[\frac{p-1}{p-2}+\frac{p-1}{p} \frac{1}{\gamma^{p}} \tilde{w}_{j}(y)+\left(\frac{p-1}{p}\right)^{2} \frac{1}{\gamma^{2 p}} \tilde{w}_{1 j}(y)\right. \\
& \left.+\left(\frac{p-1}{p}\right)^{3} \frac{1}{\gamma^{3 p}} \tilde{w}_{2 j}(y)+\frac{p-1}{p} \frac{1}{\gamma^{p}} \theta(y)\right] \\
& \times e^{\tilde{w}_{j}(y)} e^{\frac{p-1}{p} \frac{1}{\gamma^{p}} \tilde{w}_{1 j}(y)} e^{\left(\frac{p-1}{p}\right)^{2} \frac{1}{\gamma^{2 p}} \tilde{w}_{2 j}(y)} e^{\theta(y)}
\end{aligned}
$$

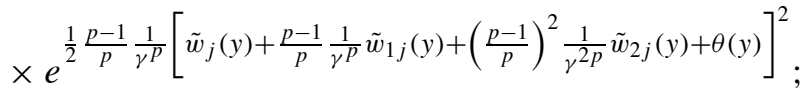

and

$$
\begin{aligned}
& I_{b}=\left(1+\frac{1}{p \gamma^{p}}\left(\tilde{w}_{j}(y)+\frac{p-1}{p} \frac{1}{\gamma^{p}} \tilde{w}_{1 j}(y)\right.\right. \\
& \left.\left.+\left(\frac{p-1}{p}\right)^{2} \frac{1}{\gamma^{2 p}} \tilde{w}_{2 j}(y)+\theta(y)\right)\right)^{2(p-1)} \\
& \times e^{\gamma^{p}\left[\left(1+\frac{1}{p \gamma^{p}}\left(\tilde{w}_{j}(y)+\frac{p-1}{p} \frac{1}{\gamma^{p}} \tilde{w}_{1 j}(y)+\left(\frac{p-1}{p}\right)^{2} \frac{1}{\gamma^{2 p}} \tilde{w}_{2 j}(y)+\theta(y)\right)\right)^{p}-1\right]} \\
& =\left[1+\frac{2(p-1)}{p} \frac{1}{\gamma^{p}} \tilde{w}_{j}(y)+2\left(\frac{p-1}{p}\right)^{2} \frac{1}{\gamma^{2 p}} \tilde{w}_{1 j}(y)\right. \\
& \left.+2\left(\frac{p-1}{p}\right)^{3} \frac{1}{\gamma^{3 p}} \tilde{w}_{2 j}(y)+\frac{2(p-1)}{p} \frac{1}{\gamma^{p}} \theta(y)\right] \\
& \times e^{\tilde{w}_{j}(y)} e^{\frac{p-1}{p} \frac{1}{\gamma^{p}} \tilde{w}_{1 j}(y)} e^{\left(\frac{p-1}{p}\right)^{2} \frac{1}{\gamma^{2 p}} \tilde{w}_{2 j}(y)} e^{\theta(y)} \\
& \times e^{\frac{1}{2} \frac{p-1}{p} \frac{1}{\gamma^{p}}\left[\tilde{w}_{j}(y)+\frac{p-1}{p} \frac{1}{\gamma^{p}} \tilde{w}_{1 j}(y)+\left(\frac{p-1}{p}\right)^{2} \frac{1}{\gamma^{2 p}} \tilde{w}_{2 j}(y)+\theta(y)\right]^{2} .}
\end{aligned}
$$


By the definition of $\tilde{w}_{1 j}$ and $\tilde{w}_{2 j}$ we get

$$
I_{a} 1_{B\left(\xi_{j}^{\prime}, R_{\varepsilon}\right)}=\frac{O(1)}{|\log \varepsilon|}, \quad I_{b} 1_{B\left(\xi_{j}^{\prime}, R_{\varepsilon}\right)}-e^{\tilde{w}_{j}(y)}=\frac{O(1)}{|\log \varepsilon|} .
$$

Finally, in the remaining region, namely where for any $j=1, \ldots, k$ we have $R_{\varepsilon}<$ $\left|y-\xi_{j}^{\prime}\right|<\frac{\delta}{\varepsilon}$, we have

$$
\left|I_{a}\right| \leq \frac{C}{|\log \varepsilon|} e^{\tilde{w}_{j}(y)}, \quad\left|I_{b}\right| \leq C e^{\tilde{w}_{j}(y)} .
$$

Then, from (2.41) and the definition of $*-$ norm, we find that very close to the point $\xi_{j}$ on $\partial \Omega$, we have

$$
\left\|f^{\prime}\left(V_{\lambda}\right)-e^{\tilde{w}_{j}}\right\|_{*, \partial \Omega_{\varepsilon}}=\frac{O(1)}{|\log \varepsilon|}
$$

which implies (2.38). Combining (2.40), (2.41) with (2.42) we obtain the estimate (2.39).

Lemma 2.7. We have

$$
\left\|f^{\prime \prime}\left(V_{\lambda}\right)\right\|_{*, \partial \Omega_{\varepsilon}} \leq C
$$

for some positive constant.

Proof. We have

$$
\begin{aligned}
f^{\prime \prime}\left(V_{\lambda}\right)= & \frac{(p-1)(p-2)}{p^{2}} \frac{1}{\gamma^{2 p}}\left(1+\frac{V_{\lambda}}{p \gamma^{p}}\right)^{p-3} e^{\gamma^{p}\left[\left(1+\frac{V_{\lambda}}{p \gamma^{p}}\right)^{p}-1\right]} \\
& +\frac{3(p-1)}{p} \frac{1}{\gamma^{p}}\left(1+\frac{V_{\lambda}}{p \gamma^{p}}\right)^{2 p-3} e^{\gamma^{p}\left[\left(1+\frac{V_{\lambda}}{p \gamma^{p}}\right)^{p}-1\right]} \\
& +\left(1+\frac{V_{\lambda}}{p \gamma^{p}}\right)^{3(p-1)} e^{\gamma^{p}\left[\left(1+\frac{V_{\lambda}}{p \gamma^{p}}\right)^{p}-1\right]}=I_{c}+I_{d}+I_{e},
\end{aligned}
$$

by a similar computation as before. Far away from the points $\xi_{j}$, namely for $\mid x-$ $\xi_{j} \mid>\delta$, i.e. $\left|y-\xi_{j}^{\prime}\right|>\frac{\delta}{\varepsilon}$, for all $j=1, \ldots, k$, we have

$I_{c}=\frac{\varepsilon^{\frac{2}{p}}}{|\log \varepsilon|^{p-1}} O(1), \quad I_{d}=\frac{\varepsilon^{\frac{2}{p}}}{|\log \varepsilon|^{2(p-1)}} O(1), \quad$ and $I_{e}=\frac{\varepsilon^{\frac{2}{p}}}{|\log \varepsilon|^{3(p-1)}} O(1)$.

Then

$$
f^{\prime \prime}\left(V_{\lambda}\right) 1_{\text {outer }}=\frac{\varepsilon^{\frac{2}{p}}}{|\log \varepsilon|^{p-1}} O(1),
$$


where again $O(1)$ denotes a function which is uniformly bounded, as $\varepsilon \rightarrow 0$, in the considered region. Let us now fix the index $j$ in $\{1, \ldots, k\}$; for $\left|y-\xi_{j}^{\prime}\right|<R_{\varepsilon}$ with any $R_{\varepsilon}:=R|\log \varepsilon|$ for some $R>0$ large but fixed, by the Taylor expansion, we have

$$
\begin{aligned}
& I_{c}=\frac{(p-1)(p-2)}{p^{2}} \frac{1}{\gamma^{2 p}}\left(1+\frac{1}{p \gamma^{p}}\left(\tilde{w}_{j}(y)+\frac{p-1}{p} \frac{1}{\gamma^{p}} \tilde{w}_{1 j}(y)\right.\right. \\
& \left.\left.+\left(\frac{p-1}{p}\right)^{2} \frac{1}{\gamma^{2 p}} \tilde{w}_{2 j}(y)+\theta(y)\right)\right)^{p-3} \\
& \times e^{\gamma^{p}\left[\left(1+\frac{1}{p \gamma^{p}}\left(\tilde{w}_{j}(y)+\frac{p-1}{p} \frac{1}{\gamma^{p}} \tilde{w}_{1 j}(y)+\left(\frac{p-1}{p}\right)^{2} \frac{1}{\gamma^{2 p}} \tilde{w}_{2 j}(y)+\theta(y)\right)\right)^{p}-1\right]} \\
& =\frac{(p-2)(p-3)}{p^{2}} \frac{1}{\gamma^{2 p}}\left[\frac{p-1}{p-3}+\frac{p-1}{p} \frac{1}{\gamma^{p}} \tilde{w}_{j}(y)+\frac{(p-1)^{2}}{p^{2}} \frac{1}{\gamma^{2 p}} \tilde{w}_{1 j}(y)\right. \\
& \left.+\frac{(p-1)^{3}}{p^{3}} \frac{1}{\gamma^{3 p}} \tilde{w}_{2 j}(y)+\frac{p-1}{p} \frac{1}{\gamma^{p}} \theta(y)\right] \\
& \times e^{\tilde{w}_{j}(y)} e^{\frac{p-1}{p} \frac{1}{\gamma^{p}} \tilde{w}_{1 j}(y)} e^{\left(\frac{p-1}{p}\right)^{2} \frac{1}{\gamma^{2 p}} \tilde{w}_{2 j}(y)} e^{\theta(y)}
\end{aligned}
$$

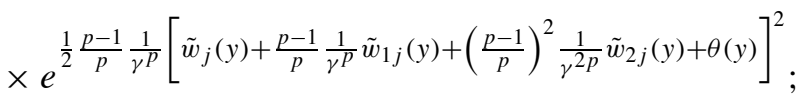

$$
\begin{aligned}
& I_{d}=\frac{3(p-1)}{p} \frac{1}{\gamma^{p}}\left(1+\frac{1}{p \gamma^{p}}\left(\tilde{w}_{j}(y)+\frac{p-1}{p} \frac{1}{\gamma^{p}} \tilde{w}_{1 j}(y)\right.\right. \\
& \left.\left.+\left(\frac{p-1}{p}\right)^{2} \frac{1}{\gamma^{2 p}} \tilde{w}_{2 j}(y)+\theta(y)\right)\right)^{2 p-3} \\
& \times e^{\gamma^{p}\left[\left(1+\frac{1}{p \gamma^{p}}\left(\tilde{w}_{j}(y)+\frac{p-1}{p} \frac{1}{\gamma^{p}} \tilde{w}_{1 j}(y)+\left(\frac{p-1}{p}\right)^{2} \frac{1}{\gamma^{2 p}} \tilde{w}_{2 j}(y)+\theta(y)\right)\right)^{p}-1\right]} \\
& =\frac{3(2 p-3)}{p} \frac{1}{\gamma^{p}}\left[\frac{p-1}{2 p-3}+\frac{p-1}{p} \frac{1}{\gamma^{p}} \tilde{w}_{j}(y)+\frac{(p-1)^{2}}{p^{2}} \frac{1}{\gamma^{2 p}} \tilde{w}_{1 j}(y)\right. \\
& \left.+\frac{(p-1)^{3}}{p^{3}} \frac{1}{\gamma^{3 p}} \tilde{w}_{2 j}(y)+\frac{p-1}{p} \frac{1}{\gamma^{p}} \theta(y)\right] \\
& \times e^{\tilde{w}_{j}(y)} e^{\frac{p-1}{p} \frac{1}{\gamma^{p}} \tilde{w}_{1 j}(y)} e^{\left(\frac{p-1}{p}\right)^{2} \frac{1}{\gamma^{2 p}} \tilde{w}_{2 j}(y)} e^{\theta(y)}
\end{aligned}
$$

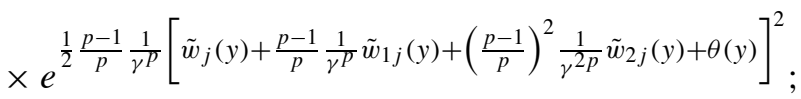


and

$$
\begin{aligned}
& I_{e}=\left(1+\frac{1}{p \gamma^{p}}\left(\tilde{w}_{j}(y)+\frac{p-1}{p} \frac{1}{\gamma^{p}} \tilde{w}_{1 j}(y)\right.\right. \\
& \left.\left.+\left(\frac{p-1}{p}\right)^{2} \frac{1}{\gamma^{2 p}} \tilde{w}_{2 j}(y)+\theta(y)\right)\right)^{3(p-1)} \\
& \times e^{\gamma^{p}\left[\left(1+\frac{1}{p \gamma^{p}}\left(\tilde{w}_{j}(y)+\frac{p-1}{p} \frac{1}{\gamma^{p}} \tilde{w}_{1 j}(y)+\left(\frac{p-1}{p}\right)^{2} \frac{1}{\gamma^{2 p}} \tilde{w}_{2 j}(y)+\theta(y)\right)\right)^{p}-1\right]} \\
& =\left[1+\frac{3(p-1)}{p} \frac{1}{\gamma^{p}} \tilde{w}_{j}(y)+\frac{3(p-1)^{2}}{p^{2}} \frac{1}{\gamma^{2 p}} \tilde{w}_{1 j}(y)\right. \\
& \left.+\frac{3(p-1)^{3}}{p^{3}} \frac{1}{\gamma^{3 p}} \tilde{w}_{2 j}(y)+\frac{3(p-1)}{p} \frac{1}{\gamma^{p}} \theta(y)\right] \\
& \times e^{\tilde{w}_{j}(y)} e^{\frac{p-1}{p} \frac{1}{\gamma^{p}} \tilde{w}_{1 j}(y)} e^{\left(\frac{p-1}{p}\right)^{2} \frac{1}{\gamma^{2 p}} \tilde{w}_{2 j}(y)} e^{\theta(y)}
\end{aligned}
$$

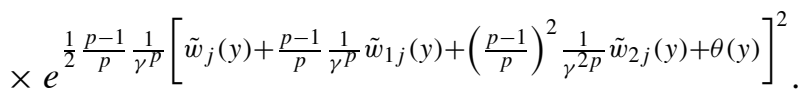

Therefore, we get

$$
I_{C} 1_{B\left(\xi_{j}^{\prime}, R_{\varepsilon}\right)}=\frac{O(1)}{|\log \varepsilon|}, \quad I_{d} 1_{B\left(\xi_{j}^{\prime}, R_{\varepsilon}\right)}=\frac{O(1)}{|\log \varepsilon|^{2}}, \quad I_{e} 1_{B\left(\xi_{j}^{\prime}, R_{\varepsilon}\right)}=O(1) .
$$

Finally, for $R_{\varepsilon}<\left|y-\xi_{j}^{\prime}\right|<\frac{\delta}{\varepsilon}$, for any $j$, we have

$$
\left|I_{c}\right| \leq \frac{C}{|\log \varepsilon|}, \quad\left|I_{d}\right| \leq \frac{C}{|\log \varepsilon|^{2}}, \quad\left|I_{e}\right|=O(1)+C e^{\tilde{w}_{j}(y)} .
$$

From (2.44) and (2.45) with (2.46), by the definition of $*$-norm, we obtain that (2.43) holds.

\section{The linearized problem}

In this section we prove the bounded invertibility of the operator $L$. First of all, we will solve the following linear problem: given $h \in C\left(\partial \Omega_{\varepsilon}\right)$, find a function $\phi$ such that

$$
\begin{cases}-\Delta \phi+\varepsilon^{2} \phi=0 & \text { in } \Omega_{\varepsilon} \\ L(\phi)=h+\sum_{j=1}^{k} c_{j} \chi_{j} Z_{1 j} & \text { on } \partial \Omega_{\varepsilon} \\ \int_{\Omega_{\varepsilon}} \chi_{j} Z_{1 j} \phi=0 & \text { for } j=1, \ldots, k\end{cases}
$$


for certain scalars $c_{j}$, where the operator $L$ is defined as in (2.31), and $Z_{1 j}, \chi_{j}$ are defined as follows: set

$$
F_{j}^{\varepsilon}(y)=\frac{1}{\varepsilon} F_{j}(\varepsilon y)
$$

with $F_{j}$ given by $(2.17)$; then

$$
Z_{i j}(y)=z_{i j}\left(F_{j}^{\varepsilon}(y)\right), \quad i=0,1, \quad j=1, \ldots, k,
$$

with $z_{0 j}$ and $z_{1 j}$ defined in (2.7) and (2.8) respectively.

Next, let us consider a large but fixed number $R_{0}>0$ and a non-negative radial and smooth cut-off function $\chi$ with $\chi(r)=1$ if $r<R_{0}$ and $\chi(r)=0$ if $r>R_{0}+1$, $0 \leq \chi \leq 1$. Then set

$$
\chi_{j}(y)=\chi\left(\left|F_{j}^{\varepsilon}(y)\right|\right) .
$$

Equation (3.1) is solved in the following proposition.

Proposition 3.1. Let $\delta>0$ be a small but fixed number, assume (2.2) and (2.3), and let $\mu_{j}$ be given by (2.28). Then there exist positive numbers $\lambda_{0}$ and $C$ such that problem (3.1) has a unique solution $\phi=T_{\lambda}(h)$ which satisfies

$$
\|\phi\|_{L^{\infty}\left(\Omega_{\varepsilon}\right)} \leq C\left(\log \frac{1}{\varepsilon}\right)\|h\|_{*, \partial \Omega_{\varepsilon}}
$$

for all $\lambda<\lambda_{0}$.

We carry out the proof in the following steps.

Step 1: Constructing a suitable barrier.

Lemma 3.2. There exist positive constants $R_{1}$ and $C$, independent of $\lambda$, such that if $\lambda$ is small enough, there exists $\psi: \Omega_{\varepsilon} \backslash \cup_{j=1}^{k} B_{R_{1}}\left(\xi_{j}^{\prime}\right) \rightarrow \mathbb{R}$, smooth and positive, satisfying

$$
\begin{cases}-\Delta \psi+\varepsilon^{2} \psi \geq \sum_{j=1}^{k} \frac{1}{\left|y-\xi_{j}^{\prime}\right|^{2+\sigma}}+\varepsilon^{2} & \text { in } \Omega_{\varepsilon} \backslash \cup_{j=1}^{k} B_{R_{1}}\left(\xi_{j}^{\prime}\right) \\ \frac{\partial \psi}{\partial \nu}-f^{\prime}\left(V_{\lambda}\right) \psi \geq \sum_{j=1}^{k} \frac{1}{\left|y-\xi_{j}^{\prime}\right|^{1+\sigma}}+\varepsilon & \text { on } \partial \Omega_{\varepsilon} \backslash \cup_{j=1}^{k} B_{R_{1}}\left(\xi_{j}^{\prime}\right) \\ \psi \geq 1 & \text { on } \Omega_{\varepsilon} \cap\left(\cup_{j=1}^{k} \partial B_{R_{1}}\left(\xi_{j}^{\prime}\right)\right) .\end{cases}
$$

Moreover, we have a uniform bound

$$
0<\psi \leq C \quad \text { in } \Omega_{\varepsilon} \backslash \cup_{j=1}^{k} B_{R_{1}}\left(\xi_{j}^{\prime}\right)
$$


Proof. Let $\eta_{j} \in C_{0}^{\infty}\left(\mathbb{R}^{2}\right)$ be such that $0 \leq \eta_{j} \leq 1, \eta_{j} \equiv 1$ in $\Omega_{\varepsilon} \cap B_{\delta / 2 \varepsilon}\left(\xi_{j}^{\prime}\right), \eta_{j} \equiv 0$ in $\Omega_{\varepsilon} \backslash B_{\delta / \varepsilon}\left(\xi_{j}^{\prime}\right),\left|\nabla \eta_{j}\right| \leq C \varepsilon$ in $\Omega_{\varepsilon},\left|\Delta \eta_{j}\right| \leq C \varepsilon^{2}$ in $\Omega$. Let $\psi_{0}(y)=\tilde{\psi}(\varepsilon y)$, where $\tilde{\psi}$ is the solution to

$$
\begin{cases}-\Delta \tilde{\psi}+\tilde{\psi}=1 & \text { in } \Omega \\ \frac{\partial \tilde{\psi}}{\partial v}=1 & \text { on } \partial \Omega\end{cases}
$$

so that

$$
-\Delta \psi_{0}+\varepsilon^{2} \psi=\varepsilon^{2} \quad \text { in } \Omega_{\varepsilon}, \quad \text { and } \frac{\partial \psi_{0}}{\partial v}=\varepsilon \quad \text { on } \partial \Omega_{\varepsilon} .
$$

In particular, $\psi_{0}$ is uniformly bounded in $\Omega_{\varepsilon}$. Take the function

$$
\psi=\sum_{j=1}^{k} \eta_{j}\left[\frac{\left(y-\xi_{j}^{\prime}\right) \cdot v\left(\xi_{j}^{\prime}\right)}{r^{1+\sigma}}+C \frac{1}{r^{\sigma}}\right]+C \psi_{0},
$$

where $r=\left|y-\xi_{j}^{\prime}-\mu_{j} v\left(\xi_{j}^{\prime}\right)\right|$. It is directly checked that $\psi$ satisfies the required condition.

Step 2: Transferring a linear equation.

We first study the linear equation

$$
\begin{cases}-\Delta \phi+\varepsilon^{2} \phi=h_{1} & \text { in } \Omega_{\varepsilon} \\ \frac{\partial \phi}{\partial v}-f^{\prime}\left(V_{\lambda}\right) \phi=h & \text { on } \partial \Omega_{\varepsilon},\end{cases}
$$

where $h_{1}, h$ are in suitable weight spaces: we consider for $h$ the norm defined in (2.34) and for $h_{1}$

$$
\left\|h_{1}\right\|_{* *, \Omega_{\varepsilon}}:=\sup _{y \in \Omega_{\varepsilon}}\left(\sum_{j=1}^{k}\left(1+\left|y-\xi_{j}^{\prime}\right|\right)^{-2-\sigma}+\varepsilon^{2}\right)^{-1}\left|h_{1}(y)\right| .
$$

For the solution of (3.4) under some orthogonality conditions, we have an a priori estimate:

Lemma 3.3. There are $R_{0}>0$ and $\lambda_{0}>0$ such that for $0<\lambda<\lambda_{0}$ and any solution of (3.4) with the orthogonality conditions

$$
\int_{\Omega_{\varepsilon}} \chi_{j} Z_{i j} \phi=0 \quad \forall i=0,1, \quad j=1, \ldots, k,
$$

we have

$$
\|\phi\|_{L^{\infty}\left(\Omega_{\varepsilon}\right)} \leq C\left(\|h\|_{*, \partial \Omega_{\varepsilon}}+\left\|h_{1}\right\|_{* *, \Omega_{\varepsilon}}\right)
$$

where $C$ is independent of $\lambda$. 
Proof. We take $R_{0}=2 R_{1}$, with $R_{1}$ being the constant of Lemma 3.2. Thanks to the barrier $\psi$ of that lemma we deduce that the following maximum principle holds in $\Omega_{\varepsilon} \backslash \cup_{j=1}^{k} B_{R_{1}}\left(\xi_{j}^{\prime}\right)$ : if $\phi \in H^{1}\left(\Omega_{\varepsilon} \backslash \cup_{j=1}^{k} B_{R_{1}}\left(\xi_{j}^{\prime}\right)\right)$ satisfies

$$
\begin{cases}-\Delta \phi+\varepsilon^{2} \phi \geq 0 & \text { in } \Omega_{\varepsilon} \backslash \cup_{j=1}^{k} B_{R_{1}}\left(\xi_{j}^{\prime}\right) \\ \frac{\partial \phi}{\partial v}-f^{\prime}\left(V_{\lambda}\right) \phi \geq 0 & \text { on } \partial \Omega_{\varepsilon} \backslash \cup_{j=1}^{k} B_{R_{1}}\left(\xi_{j}^{\prime}\right) \\ \phi \geq 0 & \text { on } \Omega_{\varepsilon} \cap\left(\cup_{j=1}^{k} \partial B_{R_{1}}\left(\xi_{j}^{\prime}\right)\right)\end{cases}
$$

then $\phi \geq 0$ in $\Omega_{\varepsilon} \backslash \cup_{j=1}^{k} B_{R_{1}}\left(\xi_{j}^{\prime}\right)$.

Let $h_{1}, h$ be bounded and $\phi$ be a solution to (3.4) satisfying (3.6). Define the inner norm of $\phi$ as

$$
\|\phi\|_{i}=\sup _{\Omega_{\varepsilon} \cap\left(\cup_{j=1}^{k} B_{R_{1}}\left(\xi_{j}^{\prime}\right)\right)}|\phi|,
$$

and set

$$
\tilde{\phi}=C_{1} \psi\left(\|\phi\|_{i}+\|h\|_{*, \partial \Omega_{\varepsilon}}+\left\|h_{1}\right\|_{* *, \Omega_{\varepsilon}}\right)
$$

with $C_{1}$ a constant independent of $\lambda$ and $\psi$ the function given in Lemma 3.2. By the above maximum principle we deduce that $\phi \leq \tilde{\phi}$ and $-\phi \leq \tilde{\phi}$ in $\Omega_{\varepsilon} \backslash \cup_{j=1}^{k} B_{R_{1}}\left(\xi_{j}^{\prime}\right)$. Since $\psi$ is uniformly bounded, we have

$$
\|\phi\|_{L^{\infty}\left(\Omega_{\varepsilon}\right)} \leq C\left(\|\phi\|_{i}+\|h\|_{*, \partial \Omega_{\varepsilon}}+\left\|h_{1}\right\|_{* *, \Omega_{\varepsilon}}\right)
$$

for some constant $C$ independent of $\phi$ and $\lambda$.

We prove the lemma by contradiction. Assume that there exist a sequence $\lambda_{n} \rightarrow 0$, points $\xi_{1}^{n}, \ldots, \xi_{k}^{n}$ on $\partial \Omega$ satisfying (2.2) and functions $\phi_{n}, f_{n}$ and $h_{n}$ with $\left\|\phi_{n}\right\|_{L^{\infty}\left(\Omega_{\varepsilon_{n}}\right)}=1,\left\|h_{1}\right\|_{* *, \Omega_{\varepsilon_{n}}} \rightarrow 0,\|h\|_{*, \partial \Omega_{\varepsilon_{n}}} \rightarrow 0$, such that, for each $n, \phi_{n}$ solves (3.4) satisfying (3.6). By (3.8) we see that $\left\|\phi_{n}\right\|_{i}$ stays away from zero. For some of the indices, say $j$, we can assume that $\sup _{B_{R_{1}}\left(\xi_{j}^{\prime}\right)}\left|\phi_{n}\right| \geq c>0$ for all $n$. Consider $\hat{\phi}_{n}=\phi_{n}\left(z-\xi_{j}^{\prime}\right)$, and let us translate and rotate $\Omega_{\varepsilon_{n}}$ such that $\Omega_{\varepsilon_{n}}$ approaches the upper half-plane and $\xi_{j}^{\prime}=0$. Then by an elliptic estimate $\hat{\phi}_{n}$ converges uniformly on compact sets to a nontrivial solution $\hat{\phi}$ of (2.6). By Proposition $2.2 \hat{\phi}$ is a linear combination of $z_{0 j}$ and $z_{1 j}$. On the other hand, taking limit in the orthogonality relation (3.6), we find that $\int_{\mathbb{R}_{+}^{2}} \chi \hat{\phi} z_{i j}=0$ for $i=0,1$. This contradicts the fact that $\hat{\phi} \not \equiv 0$.

Step 3: Establishing an a priori estimate.

In what follows, we will establish an a priori estimate for a solution to (3.4) with the orthogonality condition $\int_{\Omega_{\varepsilon}} \chi_{j} Z_{1 j} \phi=0$ only. 
Lemma 3.4. For small enough $\lambda$, if $\phi$ is a solution of (3.4) and satisfies

$$
\int_{\Omega_{\varepsilon}} \chi_{j} Z_{1 j} \phi=0 \quad \forall j=1, \ldots, k,
$$

then there holds

$$
\|\phi\|_{L^{\infty}\left(\Omega_{\varepsilon}\right)} \leq C|\log \varepsilon|\left(\|h\|_{*, \partial \Omega_{\varepsilon}}+\left\|h_{1}\right\|_{* *, \Omega_{\varepsilon}}\right),
$$

where $C$ is independent of $\lambda$.

Proof. Let $\phi$ satisfy (3.4) and (3.9). In order to use Lemma 3.3, we will modify $\phi$ to $\tilde{\phi}$ so to satisfy all orthogonality relations with respect to $Z_{i j}$ for $i=0,1$. Let $R>R_{0}+1$ be large but fixed, $\delta>0$ be small and fixed. Set

$$
\hat{Z}_{0 j}(y)=\psi Z_{0 j}(y)
$$

where

$$
\psi(y)=\tilde{h}\left(\left|F_{j}^{\varepsilon}(y)\right|\right), \quad \tilde{h}(x)=\frac{\log (\delta / \varepsilon)-\log |x|}{\log (\delta / \varepsilon)-\log R}
$$

with $F_{j}^{\varepsilon}$ the change of variables defined in (3.2). We observe that $\tilde{h}$ is just the solution to

$$
\begin{cases}\Delta \tilde{h}=0 & \text { in } B_{\delta / \varepsilon}(0) \backslash \bar{B}_{R}(0) \\ \tilde{h}=1 & |x|=R \\ \tilde{h}=0 & |x|=\delta / \varepsilon\end{cases}
$$

Let $\bar{\eta}_{1 j}, \bar{\eta}_{2 j}$ be radial smooth cut-off functions on $\mathbb{R}^{2}$ such that

$$
\begin{aligned}
& 0 \leq \bar{\eta}_{1 j} \leq 1,\left|\nabla \bar{\eta}_{1 j}\right| \leq C \quad \text { in } \mathbb{R}^{2}, \\
& \bar{\eta}_{1 j} \equiv 1 \quad \text { in } B_{R}(0), \quad \bar{\eta}_{1 j}=0 \quad \text { in } \mathbb{R}^{2} \backslash B_{R+1}(0),
\end{aligned}
$$

and

$$
\begin{aligned}
& 0 \leq \bar{\eta}_{2 j} \leq 1,\left|\nabla \bar{\eta}_{2 j}\right| \leq C \varepsilon / \delta,\left|\nabla^{2} \bar{\eta}_{2 j}\right| \leq C \varepsilon^{2} / \delta^{2} \quad \text { in } \mathbb{R}^{2}, \\
& \bar{\eta}_{2 j} \equiv 1 \quad \text { in } B_{\frac{\delta}{4 \varepsilon}}(0), \quad \bar{\eta}_{2 j}=0 \quad \text { in } \mathbb{R}^{2} \backslash B_{\frac{\delta}{3 \varepsilon}}(0) .
\end{aligned}
$$

Now, we write

$$
\eta_{1 j}(y)=\bar{\eta}_{1 j}\left(F_{j}^{\varepsilon}(y)\right), \quad \eta_{2 j}(y)=\bar{\eta}_{2 j}\left(F_{j}^{\varepsilon}(y)\right) .
$$

Define

$$
\tilde{Z}_{0 j}=\eta_{1 j} Z_{0 j}+\left(1-\eta_{1 j}\right) \eta_{2 j} \hat{Z}_{0 j}
$$

Given $\phi$ satisfying (3.4) and (3.9), we set

$$
\tilde{\phi}=\phi+\sum_{j=1}^{k} d_{j} \tilde{Z}_{0 j}, \quad \text { where } d_{j}=-\frac{\int_{\Omega_{\varepsilon}} \chi_{j} Z_{0 j} \phi}{\int_{\Omega_{\varepsilon}} Z_{0 j}^{2} \chi_{j}} .
$$


Therefore, our result is a direct consequence of the following:

\section{Claim:}

$$
\left|d_{j}\right| \leq C|\log \varepsilon|\left(\|h\|_{*, \partial \Omega_{\varepsilon}}+\left\|h_{1}\right\|_{* *, \Omega_{\varepsilon}}\right) \quad \forall j=1, \ldots, k .
$$

First, using the notation $\widetilde{L}=-\Delta+\varepsilon^{2} I$, we observe that $\tilde{\phi}$ satisfies

$$
\begin{cases}\widetilde{L}(\tilde{\phi})=h_{1}+\sum_{j=1}^{k} d_{j} \widetilde{L}\left(\tilde{Z}_{0 j}\right) & \text { in } \Omega_{\varepsilon} \\ \frac{\partial \tilde{\phi}}{\partial v}-f^{\prime}\left(V_{\lambda}\right) \tilde{\phi}=h+\sum_{j=1}^{k} d_{j}\left(\frac{\partial \tilde{Z}_{0 j}}{\partial v}-f^{\prime}\left(V_{\lambda}\right) \tilde{Z}_{0 j}\right) & \text { on } \partial \Omega_{\varepsilon} .\end{cases}
$$

Thus, by Lemma 3.3, we have

$$
\begin{aligned}
\|\tilde{\phi}\|_{L^{\infty}\left(\Omega_{\varepsilon}\right)} \leq & C \sum_{j=1}^{k}\left|d_{j}\right|\left(\left\|\frac{\partial \tilde{Z}_{0 j}}{\partial v}-f^{\prime}\left(V_{\lambda}\right) \tilde{Z}_{0 j}\right\|_{*, \partial \Omega_{\varepsilon}}+\left\|\tilde{L}\left(\tilde{Z}_{0 j}\right)\right\|_{* *, \Omega_{\varepsilon}}\right) \\
& +C\|h\|_{*, \partial \Omega_{\varepsilon}}+C\left\|h_{1}\right\|_{* *, \Omega_{\varepsilon}} .
\end{aligned}
$$

Multiplying the first equation in (3.13) by $\tilde{Z}_{0 l}$, integrating by parts and using the second equation in (3.13), we find

$$
\begin{aligned}
& d_{l}\left[\int_{\Omega_{\varepsilon}} \tilde{L}\left(\tilde{Z}_{0 l}\right) \tilde{Z}_{0 l}+\int_{\partial \Omega_{\varepsilon}} \tilde{Z}_{0 l}\left(\frac{\partial \tilde{Z}_{0 l}}{\partial v}-f^{\prime}\left(V_{\lambda}\right) \tilde{Z}_{0 l}\right)\right] \\
& =-\int_{\partial \Omega_{\varepsilon}} h \tilde{Z}_{0 l}-\int_{\Omega_{\varepsilon}} h_{1} \tilde{Z}_{0 l}+\int_{\partial \Omega_{\varepsilon}} \tilde{\phi}\left(\frac{\partial \tilde{Z}_{0 l}}{\partial v}-f^{\prime}\left(V_{\lambda}\right) \tilde{Z}_{0 l}\right)+\int_{\Omega_{\varepsilon}} \tilde{\phi} \tilde{L}\left(\tilde{Z}_{0 l}\right) .
\end{aligned}
$$

Thus by (3.14), we deduce that

$$
\begin{aligned}
& d_{l}\left[\int_{\Omega_{\varepsilon}} \tilde{L}\left(\tilde{Z}_{0 l}\right) \tilde{Z}_{0 l}+\int_{\partial \Omega_{\varepsilon}} \tilde{Z}_{0 l}\left(\frac{\partial \tilde{Z}_{0 l}}{\partial v}-f^{\prime}\left(V_{\lambda}\right) \tilde{Z}_{0 l}\right)\right] \\
& \leq C\|h\|_{*, \partial \Omega_{\varepsilon}}+C\left\|h_{1}\right\|_{* *, \Omega_{\varepsilon}}+\|\tilde{\phi}\|_{L^{\infty}\left(\Omega_{\varepsilon}\right)}\left\|\frac{\partial \tilde{Z}_{0 l}}{\partial v}-f^{\prime}\left(V_{\lambda}\right) \tilde{Z}_{0 l}\right\|_{*, \partial \Omega_{\varepsilon}} \\
& \quad+\|\tilde{\phi}\|_{L^{\infty}\left(\Omega_{\varepsilon}\right)}\left\|\tilde{L}\left(\tilde{Z}_{0 l}\right)\right\|_{* *, \Omega_{\varepsilon}} \\
& \leq C\left(\|h\|_{*, \partial \Omega_{\varepsilon}}+\left\|h_{1}\right\|_{* *, \Omega_{\varepsilon}}\right)\left(1+\left\|\frac{\partial \tilde{Z}_{0 l}}{\partial v}-f^{\prime}\left(V_{\lambda}\right) \tilde{Z}_{0 l}\right\|_{*, \partial \Omega_{\varepsilon}}^{+}+\left\|\tilde{L}\left(\tilde{Z}_{0 l}\right)\right\|_{* *, \Omega_{\varepsilon}}\right) \\
& \quad+C \sum_{j=1}^{k}\left(\left\|\frac{\partial \tilde{Z}_{0 j}}{\partial v}-f^{\prime}\left(V_{\lambda}\right) \tilde{Z}_{0 j}\right\|_{*, \partial \Omega_{\varepsilon}}+\left\|\widetilde{L}\left(\tilde{Z}_{0 j}\right)\right\|_{* *, \Omega_{\varepsilon}}\right) .
\end{aligned}
$$


We achieve the claim by proving the following estimates: for some constant $C>0$, independent of $\lambda$,

$$
\begin{aligned}
\int_{\Omega_{\varepsilon}} \tilde{L}\left(\tilde{Z}_{0 j}\right) \tilde{Z}_{0 j}+\int_{\partial \Omega_{\varepsilon}} \tilde{Z}_{0 j}\left(\frac{\partial \tilde{Z}_{0 j}}{\partial v}-f^{\prime}\left(V_{\lambda}\right) \tilde{Z}_{0 j}\right) & \geq \frac{C}{|\log \varepsilon|}, \\
\left\|\tilde{L}\left(\tilde{Z}_{0 j}\right)\right\|_{* *, \Omega_{\varepsilon}} & \leq \frac{C}{|\log \varepsilon|}, \\
\left\|\frac{\partial \tilde{Z}_{0 j}}{\partial v}-f^{\prime}\left(V_{\lambda}\right) \tilde{Z}_{0 j}\right\|_{*, \partial \Omega_{\varepsilon}} & \leq \frac{C}{|\log \varepsilon|} .
\end{aligned}
$$

In [6] it is showed that estimates (3.17), (3.18) and (3.19) hold.

Step 4: In proving the solvability of (3.1), we may first solve the following problem: given $h \in L^{\infty}\left(\Omega_{\varepsilon}\right)$ find $\phi \in L^{\infty}\left(\Omega_{\varepsilon}\right)$ and $d_{1}, \ldots, d_{k} \in \mathbb{R}$ such that

$$
\begin{cases}-\Delta \phi+\varepsilon^{2} \phi=\sum_{j=1}^{k} d_{j} \chi_{j} Z_{1 j} & \text { in } \Omega_{\varepsilon} \\ \frac{\partial \phi}{\partial v}-f^{\prime}\left(V_{\lambda}\right) \phi=h & \text { on } \partial \Omega_{\varepsilon} \\ \int_{\Omega_{\varepsilon}} \chi_{j} Z_{1 j} \phi=0 & \text { for } j=1, \ldots, k .\end{cases}
$$

We first prove that for any $\phi, d_{1}, \ldots, d_{k}$ solution to (3.20) the bound

$$
\|\phi\|_{L^{\infty}\left(\Omega_{\varepsilon}\right)} \leq C|\log \varepsilon|\|h\|_{*, \partial \Omega_{\varepsilon}}
$$

holds. In fact, by Lemma 3.4, we have

$$
\|\phi\|_{L^{\infty}\left(\Omega_{\varepsilon}\right)} \leq C|\log \varepsilon|\left(\|h\|_{*, \partial \Omega_{\varepsilon}}+\sum_{j=1}^{k}\left|d_{j}\right|\right)
$$

and therefore it is enough to prove that $\left|d_{j}\right| \leq C\|h\|_{*, \partial \Omega_{\varepsilon}}$.

Let $\eta_{2 j}$ be the cut-off function defined in (3.11), and multiply (3.20) by $\eta_{2 l} Z_{1 l}$. Integrating by parts we get

$$
\begin{aligned}
d_{l} \int_{\Omega_{\varepsilon}} \chi_{l} Z_{1 l}^{2}= & -\int_{\partial \Omega_{\varepsilon}} h \eta_{2 l} Z_{1 l}+\int_{\partial \Omega_{\varepsilon}} \phi \frac{\partial \eta_{2 l}}{\partial v} Z_{1 l} \\
& +\int_{\partial \Omega_{\varepsilon}} \phi \eta_{2 l} Z_{1 l}\left(\frac{\partial Z_{1 l}}{\partial v}-f^{\prime}\left(V_{\lambda}\right) Z_{1 l}\right) \\
& +\int_{\Omega_{\varepsilon}} \phi\left(-\Delta\left(\eta_{2 l} Z_{1 l}\right)+\varepsilon^{2} \eta_{2 l} Z_{1 l}\right) .
\end{aligned}
$$


Since, $Z_{1 l}=O\left(\frac{1}{1+r}\right)$ and $\nabla \eta_{2 l}=O(\varepsilon)$, we have

$$
\left|\int_{\partial \Omega_{\varepsilon}} \phi \frac{\partial \eta_{2 l}}{\partial \nu} Z_{1 l}\right| \leq C \varepsilon \log \frac{1}{\varepsilon} .
$$

On the other hand, we can estimate that

$$
\frac{\partial Z_{1 l}}{\partial \nu}-f^{\prime}\left(V_{\lambda}\right) Z_{1 l}=O\left(\frac{\varepsilon}{1+r}\right)+O\left(\frac{\varepsilon^{\alpha}}{1+r^{2}}\right), \quad|y|<\frac{\delta}{\varepsilon}, \quad y \in \partial \Omega_{\varepsilon},
$$

which implies that

$$
\int_{\partial \Omega_{\varepsilon}}\left|\frac{\partial Z_{1 l}}{\partial v}-f^{\prime}\left(V_{\lambda}\right) Z_{1 l}\right|=O\left(\varepsilon^{\alpha}\right) .
$$

Moreover, we deduce that

$$
\int_{\partial \Omega_{\varepsilon}}\left|-\Delta\left(\eta_{2 l} Z_{1 l}\right)+\varepsilon^{2} \eta_{2 l} Z_{1 l}\right|=O\left(\varepsilon \log \frac{1}{\varepsilon}\right) .
$$

Thus from (3.23)-(3.25), we conclude that

$$
d_{l} \int_{\Omega_{\varepsilon}} \chi_{l} Z_{1 l}^{2} \leq C\|h\|_{*, \partial \Omega_{\varepsilon}}+C \varepsilon^{\alpha}\|\phi\|_{L^{\infty}\left(\Omega_{\varepsilon}\right)} .
$$

Combining (3.22) and (3.26) we have

$$
\left|d_{l}\right| \leq C\left(\|h\|_{*, \partial \Omega_{\varepsilon}}+C \varepsilon^{\alpha} \log \frac{1}{\varepsilon^{2}} \sum_{j=1}^{k}\left|d_{j}\right|\right) .
$$

This implies that

$$
\left|d_{l}\right| \leq C\|h\|_{*, \partial \Omega_{\varepsilon}}
$$

which proves (3.21).

Now consider the Hilbert space

$$
\mathbb{H}=\left\{\phi \in H^{1}\left(\Omega_{\varepsilon}\right): \int_{\Omega_{\varepsilon}} \chi_{j} Z_{1 j} \phi=0 \quad \forall j=1, \ldots, k\right\},
$$

endowed the norm $\|\phi\|_{H^{1}}^{2}=\int_{\Omega_{\varepsilon}}|\nabla \phi|^{2}+\varepsilon^{2} \phi^{2}$. Problem (3.20), expressed in a weak form, is equivalent to seeking $\phi \in \mathbb{H}$ such that

$$
\int_{\Omega_{\varepsilon}} \nabla \phi \nabla \psi+\varepsilon^{2} \phi \psi-\int_{\partial \Omega_{\varepsilon}} f^{\prime}\left(V_{\lambda}\right) \psi=\int_{\partial \Omega_{\varepsilon}} h \psi, \quad \text { for all } \psi \in \mathbb{H} .
$$


Fredholm's alternative guarantees unique solvability of (3.20), which is satisfied by (3.21).

Step 5: In order to solve (3.1), let $Y_{i} \in L^{\infty}\left(\Omega_{\varepsilon}\right), d_{i j} \in \mathbb{R}$ be the solution of (3.20) with $h=\chi_{i} Z_{1 i}$, that is

$$
\begin{cases}-\Delta Y_{i}+\varepsilon^{2} Y_{i}=\sum_{j=1}^{k} d_{j} \chi_{j} Z_{1 j} & \text { in } \Omega_{\varepsilon} \\ \frac{\partial Y_{i}}{\partial \nu}-f^{\prime}\left(V_{\lambda}\right) Y_{i}=\chi_{i} Z_{1 i} & \text { on } \partial \Omega_{\varepsilon} \\ \int_{\Omega_{\varepsilon}} \chi_{j} Z_{1 j} Y_{i}=0 & \text { for } j=1, \ldots, k .\end{cases}
$$

From Step 4, there is a unique solution $Y_{i} \in L^{\infty}\left(\Omega_{\varepsilon}\right)$ of (3.28), and

$$
\left\|Y_{i}\right\|_{L^{\infty}\left(\Omega_{\varepsilon}\right)} \leq C|\log \varepsilon|, \quad\left|d_{i j}\right| \leq C
$$

for some constant $C$ independent of $\lambda$.

Multiplying (3.28) by $\eta_{2 j} Z_{1 j}$, and integrates by parts, we have

$$
\begin{aligned}
d_{i j} \int_{\Omega_{\varepsilon}} \chi_{j} Z_{1 j}^{2}+\delta_{i j} \int_{\partial \Omega_{\varepsilon}} \chi_{j} Z_{1 j}^{2}= & \int_{\partial \Omega_{\varepsilon}}\left(\frac{\partial Z_{1 j}}{\partial v}-f^{\prime}\left(V_{\lambda}\right) Z_{1 j}\right) \eta_{2 j} Y_{i} \\
& +\int_{\partial \Omega_{\varepsilon}} \frac{\partial \eta_{2 j}}{\partial v} Z_{1 j} Y_{i},
\end{aligned}
$$

where $\delta_{i j}$ is Kronecker's delta. From (3.24), (3.25) and (3.27) we obtain

$$
d_{i j} \int_{\Omega_{\varepsilon}} \chi_{j} Z_{1 j}^{2}+\delta_{i j} \int_{\partial \Omega_{\varepsilon}} \chi_{j} Z_{1 j}^{2}=O\left(\varepsilon^{\alpha} \log \frac{1}{\varepsilon}\right) .
$$

Then we get

$$
d_{i j}=A \delta_{i j}+O\left(\varepsilon^{\alpha} \log \frac{1}{\varepsilon}\right)
$$

with $A>0$ is independent of $\varepsilon$. Hence the matrix $D$ with entries $d_{i j}$ in invertible for small $\varepsilon$ and $\left\|D^{-1}\right\| \leq C$ uniformly in $\varepsilon$. Then, given $h \in L^{\infty}\left(\partial \Omega_{\varepsilon}\right)$ we find $\phi_{1}$, $d_{1}, \ldots, d_{k}$, the solution to (3.28) and define

$$
\phi=\phi_{1}+\sum_{i=1}^{k} c_{i} Y_{i}
$$

where $c_{i}$ satisfies

$$
\sum_{i=1}^{k} c_{i} d_{i j}=-d_{j}, \quad \forall j=1, \ldots, k
$$


Then $\phi$ satisfies (3.1) and we have

$$
\begin{aligned}
\|\phi\|_{L^{\infty}\left(\Omega_{\varepsilon}\right)} & \leq\left\|\phi_{1}\right\|_{L^{\infty}\left(\Omega_{\varepsilon}\right)}+\log \frac{1}{\varepsilon} \sum_{i=1}^{k}\left|c_{i}\right| \\
& \leq C \log \frac{1}{\varepsilon}\|h\|_{*, \partial \Omega_{\varepsilon}}+\log \frac{1}{\varepsilon} \sum_{i=1}^{k}\left|d_{i}\right| \\
& \leq C \log \frac{1}{\varepsilon}\|h\|_{*, \partial \Omega_{\varepsilon}}
\end{aligned}
$$

by (3.27). This finishes the proof of Proposition 3.1 .

Remark 3.5. A slight modification of the proof above also shows that for any $h \in$ $L^{\infty}\left(\partial \Omega_{\varepsilon}\right)$ and $h_{1} \in L^{\infty}\left(\Omega_{\varepsilon}\right)$, the equation

$$
\begin{cases}-\Delta \phi+\varepsilon^{2} \phi=h_{1} & \text { in } \Omega_{\varepsilon} \\ L(\phi)=h+\sum_{j=1}^{k} c_{j} \chi_{j} Z_{1 j} & \text { on } \partial \Omega_{\varepsilon} \\ \int_{\Omega_{\varepsilon}} \chi_{j} Z_{1 j} \phi=0 & \text { for } j=1, \ldots, k\end{cases}
$$

has a unique solution $\phi, c_{1}, \ldots, c_{k}$ satisfying

$$
\begin{aligned}
\|\phi\|_{L^{\infty}\left(\Omega_{\varepsilon}\right)} & \leq C \log \frac{1}{\varepsilon}\left(\|h\|_{*, \partial \Omega_{\varepsilon}}+\left\|h_{1}\right\|_{* *, \Omega_{\varepsilon}}\right), \\
\left|c_{j}\right| & \leq C\left(\|h\|_{*, \partial \Omega_{\varepsilon}}+\left\|h_{1}\right\|_{* *, \Omega_{\varepsilon}}\right), \quad \forall j=1, \ldots, k
\end{aligned}
$$

for some $C$ independent of $\lambda$.

Proposition 3.1 implies that the unique solution $\phi=T_{\lambda}(h)$ of (3.1) defines a continuous linear map from the Banach space $\mathcal{C}_{*}$ of all functions $h$ in $L^{\infty}$ for which $\|h\|_{*}<\infty$ into $L^{\infty}$, with norm bounded uniformly in $\lambda$.

Lemma 3.6. The operator $T_{\lambda}$ is differentiable with respect to the variables $\xi_{1}, \ldots, \xi_{k}$ on $\partial \Omega$ satisfying (2.2), and one has the estimate

$$
\left\|\partial_{\xi_{l}} T_{\lambda}(h)\right\|_{L^{\infty}\left(\Omega_{\varepsilon}\right)} \leq C\left(\log \frac{1}{\varepsilon}\right)^{2}\|h\|_{*, \partial \Omega_{\varepsilon}} \quad \text { for } l=1, \ldots, k,
$$

for a given positive $C$, independent of $\lambda$, and for all small enough $\lambda$.

Proof. Differentiating equation (3.1), formally $Z:=\partial_{\xi_{l}^{\prime}} \phi$ should satisfy in $\Omega_{\varepsilon}$ the equation

$$
-\Delta Z+\varepsilon^{2} Z=0 \quad \text { in } \Omega_{\varepsilon},
$$


and on the boundary $\partial \Omega_{\varepsilon}$

$$
L(Z)=-\partial_{\xi_{l}^{\prime}}\left(f^{\prime}\left(V_{\lambda}\right)\right) \phi+c_{l} \partial_{\xi_{l}^{\prime}}\left(\chi_{l} Z_{1 l}\right)+\sum_{j=1}^{k} d_{j} Z_{1 j} \chi_{j}
$$

with $d_{j}=\partial_{\xi_{l}^{\prime}} c_{j}$; moreover the orthogonality conditions now become

$$
\begin{aligned}
& \int_{\Omega_{\varepsilon}} Z_{1 j} \chi_{j} Z=0 \quad \text { if } j \neq l \\
& \int_{\Omega_{\varepsilon}} Z_{1 l} \chi_{l} Z=-\int_{\Omega_{\varepsilon}} \partial_{\xi_{l}^{\prime}}\left(Z_{1 l} \chi_{l}\right) \phi .
\end{aligned}
$$

We consider the constants $b_{l}$ defined by

$$
b_{l} \int_{\Omega_{\varepsilon}} \chi_{l}^{2} \mid Z_{1 l}^{2}=\int_{\Omega_{\varepsilon}} \partial_{\xi_{l}^{\prime}}\left(Z_{1 l} \chi_{l}\right) \phi, \quad \text { for } l=1, \ldots, k \text {. }
$$

Define

$$
\tilde{Z}=Z+b_{l} \chi_{l} Z_{1 l}
$$

We then have

$$
\begin{cases}-\Delta \tilde{Z}+\varepsilon^{2} \tilde{Z}=a & \text { in } \Omega_{\varepsilon} \\ L(\tilde{Z})=b+\sum_{j=1}^{k} d_{j} Z_{1 j} \chi_{j} & \text { on } \partial \Omega_{\varepsilon} \\ \int_{\Omega_{\varepsilon}} \chi_{j} Z_{1 j} \tilde{Z}=0 & \text { for } j=1, \ldots, k\end{cases}
$$

where

$$
\begin{aligned}
& a=b_{l}\left(-\Delta\left(\chi_{l} Z_{1 l}\right)+\varepsilon^{2} \chi_{l} Z_{1 l}\right), \\
& b=-\partial_{\xi_{l}^{\prime}}\left(f^{\prime}\left(V_{\lambda}\right)\right) \phi+c_{l} \partial_{\xi_{l}^{\prime}}\left(Z_{1 l} \chi_{l}\right)+L\left(\chi_{l} Z_{1 l}\right),
\end{aligned}
$$

and we have

$$
\|a\|_{* *, \Omega_{\varepsilon}} \leq C \log \frac{1}{\varepsilon}\|h\|_{*, \partial \Omega_{\varepsilon}}, \quad\|b\|_{*, \partial \Omega_{\varepsilon}} \leq C \log \frac{1}{\varepsilon}\|h\|_{*, \partial \Omega_{\varepsilon}} .
$$

Hence, using Proposition 3.1 we obtain

$$
\left\|\partial_{\xi_{l}} T_{\lambda}(h)\right\|_{L^{\infty}\left(\Omega_{\varepsilon}\right)} \leq C\left(\log \frac{1}{\varepsilon}\right)^{2}\|h\|_{*, \partial \Omega_{\varepsilon}} \quad \text { for } l=1, \ldots, k
$$




\section{The nonlinear problem}

Let us now introduce the following auxiliary nonlinear problem

$$
\begin{cases}-\Delta \phi+\varepsilon^{2} \phi=0 & \text { in } \Omega_{\varepsilon} \\ L(\phi)=E_{\lambda}+N(\phi)+\sum_{j=1}^{k} c_{j} \chi_{j} Z_{1 j} & \text { on } \partial \Omega_{\varepsilon} \\ \int_{\Omega_{\varepsilon}} \chi_{j} Z_{1 j} \phi=0 & \text { for } j=1, \ldots, k .\end{cases}
$$

We have the following result:

Proposition 4.1. Under the condition of Proposition 3.1, there exist positive numbers $\lambda_{0}$ and $C$ such that problem (4.1) has a unique solution $\phi$ which satisfies

$$
\|\phi\|_{L^{\infty}\left(\Omega_{\varepsilon}\right)} \leq \frac{C}{|\log \varepsilon|^{2}}
$$

for all $\lambda<\lambda_{0}$. Moreover, if we consider the map $\xi^{\prime} \mapsto \phi$ into the space $C\left(\bar{\Omega}_{\varepsilon}\right)$, the derivative $D_{\xi^{\prime}} \phi$ exists and defines a continuous function of $\xi^{\prime}$. Besides, there is a constant $C>0$ such that

$$
\left\|D_{\xi^{\prime}} \phi\right\|_{L^{\infty}\left(\Omega_{\varepsilon}\right)} \leq \frac{C}{|\log \varepsilon|}
$$

Proof. In terms of the operator $T_{\lambda}$ defined in Proposition 3.1, problem (4.1) becomes

$$
\phi=T_{\lambda}\left(N(\phi)+E_{\lambda}\right):=A(\phi) .
$$

For a given number $M>0$, let us consider the region

$$
\mathcal{F}_{M}:=\left\{\phi \in C\left(\bar{\Omega}_{\varepsilon}\right):\|\phi\|_{L^{\infty}\left(\Omega_{\varepsilon}\right)} \leq \frac{M}{|\log \varepsilon|^{2}}\right\} .
$$

From Proposition 3.1 we get

$$
\|A(\phi)\|_{L^{\infty}\left(\Omega_{\varepsilon}\right)} \leq C\left(\log \frac{1}{\varepsilon}\right)\left[\|N(\phi)\|_{*, \partial \Omega_{\varepsilon}}+\left\|E_{\lambda}\right\|_{*, \partial \Omega_{\varepsilon}}\right] .
$$

From (2.35) and (2.43), by the definition of $N(\phi)$ in (2.33), we have

$$
\|A(\phi)\|_{L^{\infty}\left(\Omega_{\varepsilon}\right)} \leq C|\log \varepsilon|\left(C\|\phi\|_{L^{\infty}\left(\Omega_{\varepsilon}\right)}^{2}+\frac{1}{|\log \varepsilon|^{3}}\right) .
$$


We then get that $A\left(\mathcal{F}_{M}\right) \subset \mathcal{F}_{M}$ for a sufficiently large but fixed $M$ and all small $\lambda$. Moreover, for any $\phi_{1}, \phi_{2} \in \mathcal{F}_{M}$, one has

$$
\left\|N\left(\phi_{1}\right)-N\left(\phi_{2}\right)\right\|_{*, \partial \Omega_{\varepsilon}} \leq C\left(\max _{i=1,2}\left\|\phi_{i}\right\|_{L^{\infty}\left(\Omega_{\varepsilon}\right)}\right)\left\|\phi_{1}-\phi_{2}\right\|_{L^{\infty}\left(\Omega_{\varepsilon}\right)} .
$$

In fact,

$$
\begin{aligned}
N\left(\phi_{1}\right)-N\left(\phi_{2}\right) & =f\left(V_{\lambda}+\phi_{1}\right)-f\left(V_{\lambda}+\phi_{2}\right)-f^{\prime}\left(V_{\lambda}\right)\left(\phi_{1}-\phi_{2}\right) \\
& =\int_{0}^{1}\left(\frac{d}{d t} f\left(V_{\lambda}+\phi_{2}+t\left(\phi_{1}-\phi_{2}\right)\right) d t-f^{\prime}\left(V_{\lambda}\right)\left(\phi_{1}-\phi_{2}\right)\right. \\
& =\int_{0}^{1}\left(f^{\prime}\left(V_{\lambda}+\phi_{2}+t\left(\phi_{1}-\phi_{2}\right)-f^{\prime}\left(V_{\lambda}\right)\right) d t\left(\phi_{1}-\phi_{2}\right) .\right.
\end{aligned}
$$

Thus, for a certain $t^{*} \in(0,1)$, and $s \in(0,1)$

$$
\begin{aligned}
& \left|N\left(\phi_{1}\right)-N\left(\phi_{2}\right)\right| \leq C \mid f^{\prime}\left(V_{\lambda}+\phi_{2}+t^{*}\left(\phi_{1}-\phi_{2}\right)-f^{\prime}\left(V_{\lambda}\right) \mid\left\|\phi_{1}-\phi_{2}\right\|_{L^{\infty}\left(\Omega_{\varepsilon}\right)}\right. \\
& \leq C\left|f^{\prime \prime}\left(V_{\lambda}+s \phi_{2}+t^{*}\left(\phi_{1}-\phi_{2}\right)\right)\right|\left(\left\|\phi_{1}\right\|_{L^{\infty}\left(\Omega_{\varepsilon}\right)}+\left\|\phi_{2}\right\|_{L^{\infty}\left(\Omega_{\varepsilon}\right)}\right)\left\|\phi_{1}-\phi_{2}\right\|_{L^{\infty}\left(\Omega_{\varepsilon}\right)} .
\end{aligned}
$$

Thanks to (2.43) and the fact that $\left\|\phi_{1}\right\|_{L^{\infty}\left(\Omega_{\varepsilon}\right)},\left\|\phi_{2}\right\|_{L^{\infty}\left(\Omega_{\varepsilon}\right)} \rightarrow 0$ as $\lambda \rightarrow 0$, we conclude that

$$
\begin{aligned}
& \left\|N\left(\phi_{1}\right)-N\left(\phi_{2}\right)\right\|_{*, \partial \Omega_{\varepsilon}} \\
& \leq C\left\|f^{\prime \prime}\left(V_{\lambda}\right)\right\|_{*, \partial \Omega_{\varepsilon}}\left(\left\|\phi_{1}\right\|_{L^{\infty}\left(\Omega_{\varepsilon}\right)}+\left\|\phi_{2}\right\|_{L^{\infty}\left(\Omega_{\varepsilon}\right)}\right)\left\|\phi_{1}-\phi_{2}\right\|_{L^{\infty}\left(\Omega_{\varepsilon}\right)} \\
& \leq C\left(\left\|\phi_{1}\right\|_{L^{\infty}\left(\Omega_{\varepsilon}\right)}+\left\|\phi_{2}\right\|_{L^{\infty}\left(\Omega_{\varepsilon}\right)}\right)\left\|\phi_{1}-\phi_{2}\right\|_{L^{\infty}\left(\Omega_{\varepsilon}\right)} .
\end{aligned}
$$

Then we have

$$
\begin{aligned}
\left\|A\left(\phi_{1}\right)-A\left(\phi_{2}\right)\right\|_{L^{\infty}\left(\Omega_{\varepsilon}\right)} & \leq C|\log \varepsilon|\left\|N\left(\phi_{1}\right)-N\left(\phi_{2}\right)\right\|_{*, \partial \Omega_{\varepsilon}} \\
& \leq C|\log \varepsilon|\left(\max _{i=1,2}\left\|\phi_{i}\right\|_{L^{\infty}\left(\Omega_{\varepsilon}\right)}\right)\left\|\phi_{1}-\phi_{2}\right\|_{L^{\infty}\left(\Omega_{\varepsilon}\right)}
\end{aligned}
$$

Thus the operator $A$ has a small Lipschitz constant in $\mathcal{F}_{M}$ for all small $\lambda$, therefore a unique fixed point of $A$ exists in this region.

We shall next analyze the differentiability of the map $\xi^{\prime}=\left(\xi_{1}^{\prime}, \ldots, \xi_{k}^{\prime}\right) \mapsto \phi$. Assume for instance that the partial derivative $\partial_{\xi_{l}} \phi$ exists, for $l=1, \ldots, k$. Since $\phi=T_{\lambda}\left(N(\phi)+E_{\lambda}\right)$, formally we have

$$
\partial_{\xi_{l}^{\prime}} \phi=\left(\partial_{\xi_{l}^{\prime}} T_{\lambda}\right)\left(N(\phi)+E_{\lambda}\right)+T_{\lambda}\left(\partial_{\xi_{l}^{\prime}} N(\phi)+\partial_{\xi_{l}^{\prime}} E_{\lambda}\right) .
$$

From (3.31), we have

$$
\left\|\partial_{\xi_{l}} T_{\lambda}\left(N(\phi)+E_{\lambda}\right)\right\|_{L^{\infty}\left(\Omega_{\varepsilon}\right)} \leq C|\log \varepsilon|^{2}\left\|N(\phi)+E_{\lambda}\right\|_{*, \partial \Omega_{\varepsilon}} \leq \frac{C}{|\log \varepsilon|} .
$$


On the other hand,

$$
\begin{aligned}
\partial_{\xi_{m}^{\prime}} N(\phi)= & {\left[f^{\prime}\left(V_{\lambda}+\phi\right)-f^{\prime}\left(V_{\lambda}\right)-f^{\prime \prime}\left(V_{\lambda}\right) \phi\right] \partial_{\xi_{l}^{\prime}} V_{\lambda}+\partial_{\xi_{l}^{\prime}}\left[f^{\prime}\left(V_{\lambda}\right)-e^{w_{\mu_{j}}}\right] \phi } \\
& +\left[f^{\prime}\left(V_{\lambda}+\phi\right)-f^{\prime}\left(V_{\lambda}\right)\right] \partial_{\xi_{l}^{\prime}} \phi+\left[f^{\prime}\left(V_{\lambda}\right)-e^{w_{\mu_{j}}}\right] \partial_{\xi_{l}^{\prime}} \phi .
\end{aligned}
$$

Then,

$$
\begin{aligned}
\left\|\partial_{\xi_{l}^{\prime}} N(\phi)\right\|_{*, \partial \Omega_{\varepsilon}} \leq C\{ & \|\phi\|_{L^{\infty}\left(\Omega_{\varepsilon}\right)}^{2}+\frac{1}{|\log \varepsilon|}\|\phi\|_{L^{\infty}\left(\Omega_{\varepsilon}\right)} \\
& \left.+\left\|\partial_{\xi_{l}^{\prime}} \phi\right\|_{L^{\infty}\left(\Omega_{\varepsilon}\right)}\|\phi\|_{L^{\infty}\left(\Omega_{\varepsilon}\right)}+\frac{1}{|\log \varepsilon|}\left\|\partial_{\xi_{l}^{\prime}} \phi\right\|_{L^{\infty}\left(\Omega_{\varepsilon}\right)}\right\} .
\end{aligned}
$$

Since $\left\|\partial_{\xi_{l}} E_{\lambda}\right\|_{*, \partial \Omega_{\varepsilon}} \leq \frac{C}{|\log \varepsilon|^{3}}$, by Proposition 3.1 we then have

$$
\left\|\partial_{\xi_{l}^{\prime}} \phi\right\|_{L^{\infty}\left(\Omega_{\varepsilon}\right)} \leq \frac{C}{|\log \varepsilon|}
$$

for all $l=1, \ldots, k$. Hence the regularity of the map $\xi^{\prime} \mapsto \phi$ can be proved by standard arguments involving the implicit function theorem and the fixed point representation (4.4). This concludes proof of the proposition.

\section{Variational reduction}

After problem (4.1) has been solved, in order to find a solution to the original problem we need to find $\xi^{\prime}$ such that

$$
c_{j}\left(\xi^{\prime}\right)=0 \text { for all } j=1, \ldots, k .
$$

This problem is indeed variational: it is equivalent to finding the critical points of a function of $\xi=\varepsilon \xi^{\prime}$. Associated to (1.1), let us introduce the energy functional $J_{\lambda}: H^{1}(\Omega) \rightarrow \mathbb{R}$ given by

$$
J_{\lambda}(u)=\frac{1}{2} \int_{\Omega}\left(|\nabla u|^{2}+u^{2}\right)-\frac{\lambda}{p} \int_{\partial \Omega} e^{u^{p}},
$$

and the finite-dimensional restriction

$$
F_{\lambda}(\xi)=J_{\lambda}\left(U_{\lambda}(\xi)+\tilde{\phi}(\xi)\right)
$$

where $\tilde{\phi}=\tilde{\phi}(\xi)=\tilde{\phi}(x, \xi)$ is the function defined in $\Omega$ by the relation $\tilde{\phi}(x, \xi)=$ $\phi\left(\frac{x}{\varepsilon}, \frac{\xi}{\varepsilon}\right)$, with $\phi$ the unique solution to problem (4.1) given by Proposition 3.1.

Lemma 5.1. The functional $F_{\lambda}(\xi)$ is of class $C^{1}$. Moreover, for all $\lambda>0$ sufficiently small, if $D_{\xi} F_{\lambda}(\xi)=0$, then $\xi$ satisfies (5.1). 
Proof. A direct consequence of Proposition 4.1 and the definition of the function $U_{\lambda}$ is the fact that the map $\xi \mapsto F_{\lambda}(\xi)$ is of class $C^{1}$. Define

$$
I_{\lambda}(v)=\frac{1}{2} \int_{\Omega_{\varepsilon}}\left(|\nabla v|^{2}+\varepsilon^{2} v^{2}\right)-\int_{\partial \Omega_{\varepsilon}} e^{\gamma^{p}\left[\left(1+\frac{v}{p \gamma^{p}}\right)^{p}-1\right]} .
$$

Let us differentiate the function $F_{\lambda}(\xi)$ with the respect to $\xi$. Since

$$
J_{\lambda}\left(\left(U_{\lambda}+\tilde{\phi}\right)(x, \xi)\right)=\frac{1}{p^{2} \gamma^{2(p-1)}} I_{\lambda}\left(\left(V_{\lambda}+\phi\right)\left(\frac{x}{\varepsilon}, \frac{\xi}{\varepsilon}\right)\right),
$$

we can differentiate directly $I_{\lambda}\left(V_{\lambda}(\xi)+\phi(\xi)\right)$ under the integral sign, so that

$$
\begin{aligned}
\partial_{\xi_{l}} F_{\lambda}(\xi) & =\frac{1}{p^{2} \gamma^{2(p-1)}} \varepsilon^{-1} D I_{\lambda}\left(V_{\lambda}(\xi)+\phi(\xi)\right)\left[\partial_{\xi_{l}^{\prime}} V_{\lambda}(\xi)+\partial_{\xi_{l}^{\prime}} \phi(\xi)\right] \\
& =\frac{1}{p^{2} \gamma^{2(p-1)}} \varepsilon^{-1} \sum_{j=1}^{k} \int_{\partial \Omega_{\varepsilon}} c_{j} \chi_{j} Z_{1 j}\left[\partial_{\xi_{l}^{\prime}} V_{\lambda}(\xi)+\partial_{\xi_{l}^{\prime}} \phi(\xi)\right] \\
& =\frac{1}{p^{2} \gamma^{2(p-1)}} \varepsilon^{-1}\left[\sum_{j=1}^{k} \int_{\partial \Omega_{\varepsilon}} c_{j} \chi_{j} Z_{1 j} \partial_{\xi_{l}} V_{\lambda}(\xi)+\sum_{j=1}^{k} \int_{\partial \Omega_{\varepsilon}} c_{j} \chi_{j} Z_{1 j} \partial_{\xi_{l}^{\prime}} \phi(\xi)\right] .
\end{aligned}
$$

By the expansion of $V_{\lambda}$, we have

$$
\begin{aligned}
\partial_{\xi_{l}^{\prime}} V_{\lambda} & =\partial_{\xi_{l}^{\prime}}\left(\tilde{w}_{l}(y)+\frac{p-1}{p} \frac{1}{\gamma^{p}} \tilde{w}_{1 l}(\varepsilon y)+\left(\frac{p-1}{p}\right)^{2} \frac{1}{\gamma^{2 p}} \tilde{w}_{2 l}(\varepsilon y)+\theta(y)\right) \\
& =\partial_{\xi_{l}} \tilde{w}_{l}(y)+\frac{p-1}{p} \frac{1}{\gamma^{p}} \partial_{\xi_{l}} \tilde{w}_{1 l}(\varepsilon y)+\left(\frac{p-1}{p}\right)^{2} \frac{1}{\gamma^{2 p}} \partial_{\xi_{l}^{\prime}} \tilde{w}_{2 l}(\varepsilon y)+\partial_{\xi_{l}^{\prime}} \theta(y) \\
& =-Z_{1 l}+\frac{p-1}{p} \frac{1}{\gamma^{p}} \partial_{\xi_{l}^{\prime}} \tilde{w}_{1 l}(\varepsilon y)+\left(\frac{p-1}{p}\right)^{2} \frac{1}{\gamma^{2 p}} \partial_{\xi_{l}^{\prime}} \tilde{w}_{2 l}(\varepsilon y)+\partial_{\xi_{l}^{\prime}} \theta(y) .
\end{aligned}
$$

Hence, for $j \neq l$, we have

$$
\int_{\partial \Omega_{\varepsilon}} \chi_{j} Z_{1 j} \partial_{\xi_{l}^{\prime}} V_{\lambda}(\xi)=-\left(\int_{\partial \Omega_{\varepsilon}} \chi_{j} Z_{1 j} Z_{1 l}\right)\left(1+O\left(\frac{1}{|\log \varepsilon|}\right)\right)=O(\varepsilon)
$$

while for $j=l$ we have

$$
\int_{\partial \Omega_{\varepsilon}} \chi_{j} Z_{1 j} \partial_{\xi_{l}^{\prime}} V_{\lambda}(\xi)=-\left(\int_{\partial \Omega_{\varepsilon}} \chi_{l} Z_{1 l}^{2}\right)\left(1+O\left(\frac{1}{|\log \varepsilon|}\right)\right) .
$$

We thus conclude that

$$
\sum_{j=1}^{k} c_{j} \int_{\partial \Omega_{\varepsilon}} \chi_{j} Z_{1 j} \partial_{\xi_{l}} V_{\lambda}(\xi)=-c_{l}\left(\int_{\partial \Omega_{\varepsilon}} \chi_{l} Z_{1 l}^{2}\right)\left(1+O\left(\frac{1}{|\log \varepsilon|}\right)\right)+\sum_{j \neq l} c_{j} O(\varepsilon) .
$$


On the other hand, taking into account (4.3), we get

$$
\left|\sum_{j=1}^{k} c_{j} \int_{\partial \Omega_{\varepsilon}} \chi_{j} Z_{1 j} \partial_{\xi_{l}^{\prime}} \phi(\xi)\right| \leq \frac{C}{|\log \varepsilon|} \sum_{j=1}^{k}\left|c_{j}\right| .
$$

Then, if $D_{\xi} F_{\lambda}(\xi)=0$, for $j=1,2, \ldots, k$, we then have

$$
c_{l}\left(\int_{\partial \Omega_{\varepsilon}} \chi_{l} Z_{1 l}^{2}\right)\left(1+O\left(\frac{1}{|\log \varepsilon|}\right)\right)+\sum_{j \neq l} c_{j} O(\varepsilon)=0, \text { for all } l=1, \ldots, k .
$$

This concludes the proof of the lemma.

Next, we will write the expansion of $F_{\lambda}(\xi)$ as $\lambda$ goes to zero.

Lemma 5.2. Let $\delta>0$ be fixed and assume that the constants $\mu_{j}$ are given by (2.28). Then there exist positive numbers $\lambda_{0}$ and $C$ such that the following expansion holds

$$
\lambda^{-1} \varepsilon^{\frac{2-p}{p}} F_{\lambda}(\xi)=-\frac{2 k \pi}{p}+\frac{2 k \pi}{p} \log \frac{1}{\varepsilon}+\frac{\pi}{2-p} \varphi_{k}(\xi)+|\log \varepsilon|^{-1} \Theta_{\lambda}(\xi)
$$

where

$$
\varphi_{k}(\xi)=\varphi_{k}\left(\xi_{1}, \ldots, \xi_{k}\right)=-\left[\sum_{j=1}^{k} H\left(\xi_{j}, \xi_{j}\right)+\sum_{l \neq j} G\left(\xi_{l}, \xi_{j}\right)\right] .
$$

In (5.6) $\Theta_{\lambda}(\xi)$ denotes a generic smooth function, which is uniformly bounded, as $\lambda \rightarrow 0$, for points $\xi$ satisfying (2.2).

Proof. We have

$$
\begin{aligned}
F_{\lambda}(\xi) & =J_{\lambda}\left(U_{\lambda}(\xi)+\tilde{\phi}(\xi)\right) \\
& =\frac{1}{2} \int_{\Omega}\left[\left|\nabla\left(U_{\lambda}+\tilde{\phi}\right)\right|^{2}+\left(U_{\lambda}+\tilde{\phi}\right)^{2}\right]-\frac{\lambda}{p} \int_{\partial \Omega} e^{\left(U_{\lambda}+\tilde{\phi}\right)^{p}} .
\end{aligned}
$$

From (5.4) we have that

$$
J_{\lambda}\left(U_{\lambda}(\xi)+\tilde{\phi}(\xi)\right)-J_{\lambda}\left(U_{\lambda}(\xi)\right)=\frac{1}{p^{2} \gamma^{2(p-1)}}\left[I_{\lambda}\left(V_{\lambda}+\phi\right)-I_{\lambda}\left(V_{\lambda}\right)\right]
$$

Since by construction $I_{\lambda}^{\prime}\left(V_{\lambda}+\phi\right)[\phi]=0$, we have

$$
\begin{aligned}
& J_{\lambda}\left(U_{\lambda}(\xi)+\tilde{\phi}(\xi)\right)-J_{\lambda}\left(U_{\lambda}(\xi)\right)=\frac{1}{p^{2} \gamma^{2(p-1)}} \int_{0}^{1} D^{2} I_{\lambda}\left(V_{\lambda}+t \phi\right) \phi^{2}(1-t) d t \\
& =\frac{1}{p^{2} \gamma^{2(p-1)}} \int_{0}^{1}\left[\int_{\partial \Omega_{\varepsilon}}\left(E_{\lambda}+N(\phi)\right) \phi+\int_{\partial \Omega_{\varepsilon}}\left[f_{\lambda}^{\prime}\left(V_{\lambda}\right)-f_{\lambda}^{\prime}\left(V_{\lambda}+t \phi\right)\right] \phi^{2}\right](1-t) d t .
\end{aligned}
$$


Since $\left\|E_{\lambda}\right\|_{*, \partial \Omega_{\varepsilon}} \leq \frac{C}{|\log \varepsilon|^{3}},\|\phi\|_{L^{\infty}\left(\Omega_{\varepsilon}\right)} \leq \frac{C}{|\log \varepsilon|^{2}},\|N(\phi)\|_{*, \partial \Omega_{\varepsilon}} \leq \frac{C}{|\log \varepsilon|^{4}}$ and (2.43), we get that

$$
\left|J_{\lambda}\left(U_{\lambda}(\xi)+\tilde{\phi}(\xi)\right)-J_{\lambda}\left(U_{\lambda}(\xi)\right)\right| \leq \frac{C}{\gamma^{2(p-1)}|\log \varepsilon|^{3}} .
$$

Next we expand

$$
J_{\lambda}\left(U_{\lambda}(\xi)\right)=\frac{1}{2} \int_{\Omega}\left[\left|\nabla\left(U_{\lambda}(\xi)\right)\right|^{2}+U_{\lambda}(\xi)^{2}\right]-\frac{\lambda}{p} \int_{\partial \Omega} e^{\left(U_{\lambda}(\xi)\right)^{p}} .
$$

Now we write

$$
U_{j}(x):=u_{j}(x)+H_{j}^{\varepsilon}(x), \quad U_{1 j}:=\tilde{w}_{1 j}(x)+H_{1 j}^{\varepsilon}(x), \quad U_{2 j}:=\tilde{w}_{2 j}(x)+H_{2 j}^{\varepsilon}(x) .
$$

By (2.18), we have

$$
U_{\lambda}(x)=\frac{1}{p \gamma^{p-1}} \sum_{j=1}^{k}\left(U_{j}(x)+\frac{p-1}{p} \frac{1}{\gamma^{p}} U_{1 j}(x)+\left(\frac{p-1}{p}\right)^{2} \frac{1}{\gamma^{2 p}} U_{2 j}(x)\right) .
$$

Then we have

$$
\begin{aligned}
\frac{1}{2} \int_{\Omega}\left[\left|\nabla\left(U_{\lambda}(\xi)\right)\right|^{2}+U_{\lambda}(\xi)^{2}\right] & \frac{1}{p^{2} \gamma^{2(p-1)}}\left\{\frac{1}{2} \sum_{j=1}^{k} \int_{\Omega}\left(\left|\nabla U_{j}\right|^{2}+U_{j}^{2}\right)+\sum_{l \neq j} \int_{\Omega}\left(\nabla U_{l} \nabla U_{j}+U_{l} U_{j}\right)\right. \\
& +\frac{p-1}{p} \frac{1}{\gamma^{p}} \sum_{j=1}^{k} \int_{\Omega}\left(\nabla U_{j} \nabla U_{1 j}+U_{j} U_{1 j}\right) \\
& +\left(\frac{p-1}{p}\right)^{2} \frac{1}{\gamma^{2 p}} \sum_{j=1}^{k} \int_{\Omega}\left(\nabla U_{j} \nabla U_{2 j}+U_{j} U_{2 j}\right) \\
& +\left(\frac{p-1}{p}\right)^{2} \frac{1}{\gamma^{2 p}}\left[\frac{1}{2} \sum_{j=1}^{k} \int_{\Omega}\left(\left|\nabla U_{1 j}\right|^{2}+U_{1 j}^{2}\right)\right. \\
& \left.+\left(\frac{p-1}{p}\right)^{3} \frac{1}{\gamma^{3 p}} \sum_{j=1}^{k} \int_{\Omega}\left(\nabla U_{1 j}\left(\nabla U_{1 l} \nabla U_{1 j}+U_{1 l} U_{1 j}\right)\right] U_{1 j} U_{2 j}\right) \\
& +\left(\frac{p-1}{p}\right)^{4} \frac{1}{\gamma^{4 p}}\left[\frac{1}{2} \sum_{j=1}^{k} \int_{\Omega}\left(\left|\nabla U_{2 j}\right|^{2}+U_{2 j}^{2}\right)\right. \\
& \left.\left.+\sum_{l \neq j} \int_{\Omega}\left(\nabla U_{2 l} \nabla U_{2 j}+U_{2 l} U_{2 j}\right)\right]\right\} .
\end{aligned}
$$


Let us estimate the first two terms. We observe that the remaining terms are $O\left(\frac{1}{\gamma^{2(p-1)} \gamma^{p}}\right)$. We have

$$
\begin{aligned}
\int_{\Omega}\left(\left|\nabla U_{j}\right|^{2}+U_{j}^{2}\right)= & \int_{\Omega}\left|\nabla u_{j}\right|^{2}+\int_{\Omega} u_{j}^{2}+\int_{\Omega}\left|\nabla H_{j}^{\varepsilon}\right|^{2}+\int_{\Omega}\left(H_{j}^{\varepsilon}\right)^{2} \\
& +2 \int_{\Omega} \nabla u_{j} \nabla H_{j}^{\varepsilon}+2 \int_{\Omega} u_{j} H_{j}^{\varepsilon} .
\end{aligned}
$$

Multiplying (2.4) by $H_{j}^{\varepsilon}$ yields

$$
\begin{aligned}
\int_{\Omega}\left|\nabla H_{j}^{\varepsilon}\right|^{2}+\int_{\Omega}\left(H_{j}^{\varepsilon}\right)^{2} & =-\int_{\Omega} u_{j} H_{j}^{\varepsilon}+\int_{\partial \Omega} \frac{\partial H_{j}^{\varepsilon}}{\partial v} H_{j}^{\varepsilon} \\
& =-\int_{\Omega} u_{j} H_{j}^{\varepsilon}+\varepsilon \int_{\partial \Omega} e^{u_{j}} H_{j}^{\varepsilon}-\int_{\partial \Omega} \frac{\partial u_{j}}{\partial v} H_{j}^{\varepsilon} .
\end{aligned}
$$

Multiplying (2.4) by $u_{j}^{\varepsilon}$ again, we find

$$
\int_{\Omega} u_{j}^{2}+\int_{\Omega} H_{j}^{\varepsilon} u_{j}=-\int_{\Omega} \nabla u_{j} \nabla H_{j}^{\varepsilon}+\varepsilon \int_{\partial \Omega} e^{u_{j}} u_{j}-\int_{\partial \Omega} \frac{\partial u_{j}}{\partial \nu} u_{j} .
$$

Then we get

$$
\begin{aligned}
& \int_{\Omega}\left(\left|\nabla U_{j}\right|^{2}+U_{j}^{2}\right) \\
& =\int_{\Omega}\left|\nabla u_{j}\right|^{2}-\int_{\partial \Omega} \frac{\partial u_{j}}{\partial v} u_{j}+\int_{\Omega} \nabla u_{j} \nabla H_{j}^{\varepsilon}-\int_{\partial \Omega} \frac{\partial u_{j}}{\partial v} H_{j}^{\varepsilon}+\varepsilon \int_{\partial \Omega} e^{u_{j}}\left(u_{j}+H_{j}^{\varepsilon}\right) \\
& =\varepsilon \int_{\partial \Omega} e^{u_{j}}\left(u_{j}+H_{j}^{\varepsilon}\right) \\
& =\varepsilon \int_{\partial \Omega} \frac{2 \mu_{j}}{\left|x-\xi_{j}-\varepsilon \mu_{j} v\left(\xi_{j}\right)\right|^{2}}\left(\log \frac{1}{\left|x-\xi_{j}-\varepsilon \mu_{j} v\left(\xi_{j}\right)\right|^{2}}+H\left(x, \xi_{j}\right)+O\left(\varepsilon^{\alpha}\right)\right) .
\end{aligned}
$$

Taking the change of variables $y=\frac{x-\xi_{j}}{\varepsilon \mu_{j}}$, we have

$$
\begin{aligned}
& \int_{\Omega}\left(\left|\nabla U_{j}\right|^{2}+U_{j}^{2}\right) \\
& =\int_{\partial \Omega_{\varepsilon \mu_{j}}} \frac{2}{|y-v(0)|^{2}}\left(\log \frac{1}{|y-v(0)|^{2}}+H\left(\xi_{j}+\varepsilon \mu_{j} y, \xi_{j}\right)-2 \log \left(\mu_{j} \varepsilon\right)\right) \\
& \quad+O\left(\varepsilon^{\alpha}\right) .
\end{aligned}
$$

Since

$$
\begin{aligned}
\int_{\partial \Omega_{\varepsilon \mu_{j}}} \frac{1}{|y-v(0)|^{2}} & =\pi+O(\varepsilon) \\
\int_{\partial \Omega_{\varepsilon \mu_{j}}} \frac{1}{|y-v(0)|^{2}} \log \frac{1}{|y-v(0)|^{2}} & =\int_{-\infty}^{\infty} \frac{1}{1+t^{2}} \log \frac{1}{1+t^{2}} d t+O\left(\varepsilon^{\alpha}\right) \\
& =-2 \pi \log 2+O\left(\varepsilon^{\alpha}\right),
\end{aligned}
$$


and

$$
\begin{aligned}
& \int_{\partial \Omega_{\varepsilon \mu_{j}}} \frac{2}{|y-v(0)|^{2}}\left(H\left(\xi_{j}+\varepsilon \mu_{j} y, \xi_{j}\right)-H\left(\xi_{j}, \xi_{j}\right)\right) \\
& =\int_{\partial \Omega_{\varepsilon \mu_{j}}} \frac{2}{|y-v(0)|^{2}} O\left(\varepsilon^{\alpha}|y|^{\alpha}\right)=O\left(\varepsilon^{\alpha}\right)
\end{aligned}
$$

we obtain

$$
\begin{aligned}
& \frac{1}{2} \sum_{j=1}^{k} \int_{\Omega}\left(\left|\nabla U_{j}\right|^{2}+U_{j}^{2}\right) \\
& =-2 k \pi \log 2+\pi \sum_{j=1}^{k}\left[H\left(\xi_{j}, \xi_{j}\right)-2 \log \left(\varepsilon \mu_{j}\right)\right]+O\left(\varepsilon^{\alpha}\right) \\
& =-2 k \pi \log \varepsilon+\pi \sum_{j=1}^{k}\left[H\left(\xi_{j}, \xi_{j}\right)-2 \log \left(2 \mu_{j}\right)\right]+O\left(\varepsilon^{\alpha}\right) .
\end{aligned}
$$

On the other hand, we have

$$
\begin{aligned}
& \sum_{l \neq j} \int_{\Omega}\left(\nabla U_{l} \nabla U_{j}+U_{l} U_{j}\right) \\
& =\sum_{l \neq j} \int_{\Omega} \nabla u_{l} \nabla u_{j}+2 \int_{\Omega} \nabla u_{l} \nabla H_{j}^{\varepsilon}+\int_{\Omega} \nabla H_{l}^{\varepsilon} \nabla H_{j}^{\varepsilon} \\
& \quad+\int_{\Omega} u_{l} u_{j}+2 \int_{\Omega} u_{l} H_{j}^{\varepsilon}+\int_{\Omega} H_{l}^{\varepsilon} H_{j}^{\varepsilon} .
\end{aligned}
$$

Multiplying (2.4) by $H_{l}^{\varepsilon}$ yields

$$
\int_{\Omega} \nabla H_{j}^{\varepsilon} \nabla H_{l}^{\varepsilon}+\int_{\Omega} H_{j}^{\varepsilon} H_{l}^{\varepsilon}=-\int_{\Omega} u_{j} H_{l}^{\varepsilon}+\varepsilon \int_{\partial \Omega} e^{u_{j}} H_{l}^{\varepsilon}-\int_{\partial \Omega} \frac{\partial u_{j}}{\partial \nu} H_{l}^{\varepsilon} .
$$

Multiplying (2.4) by $u_{l}^{\varepsilon}$ again, we have

$$
\int_{\Omega} \nabla u_{j}^{\varepsilon} \nabla u_{l}^{\varepsilon}+\int_{\Omega} H_{j}^{\varepsilon} u_{l}^{\varepsilon}=-\int_{\Omega} \nabla H_{j}^{\varepsilon} \nabla u_{l}+\varepsilon \int_{\partial \Omega} e^{u_{j}} u_{l}^{\varepsilon}-\int_{\partial \Omega} \frac{\partial u_{j}}{\partial \nu} u_{l} .
$$

By (5.14)-(5.16) we find that

$$
\begin{aligned}
& \sum_{l \neq j} \int_{\Omega}\left(\nabla U_{l} \nabla U_{j}+U_{l} U_{j}\right) \\
& =\sum_{l \neq j} \int_{\Omega} \nabla u_{l} \nabla u_{j}+2 \int_{\Omega} \nabla u_{l} \nabla H_{j}^{\varepsilon}+\int_{\Omega} \nabla H_{l}^{\varepsilon} \nabla H_{j}^{\varepsilon} \\
& \quad+\int_{\Omega} u_{l} u_{j}+2 \int_{\Omega} u_{l} H_{j}^{\varepsilon}+\int_{\Omega} H_{l}^{\varepsilon} H_{j}^{\varepsilon} \\
& =\pi \sum_{l \neq j} G\left(\xi_{l}, \xi_{j}\right)+O\left(\varepsilon^{\alpha}\right) .
\end{aligned}
$$


Therefore, by (5.10), (5.13) and (5.17), using the choice of $\mu_{j}$ in (2.28), we get

$$
\begin{aligned}
& \frac{1}{2} \int_{\Omega}\left[\left|\nabla\left(U_{\lambda}(\xi)\right)\right|^{2}+U_{\lambda}(\xi)^{2}\right] \\
& =\frac{1}{p^{2} \gamma^{2(p-1)}}\left[-2 k \pi \log \varepsilon-\frac{p}{2-p} \pi\left(\sum_{j=1}^{k} H\left(\xi_{j}, \xi_{j}\right)+\sum_{l \neq j}^{k} G\left(\xi_{l}, \xi_{j}\right)\right)\right. \\
& \left.+O\left(|\log \varepsilon|^{-1}\right)\right] .
\end{aligned}
$$

Finally, let use estimate the second term in (5.9). We have

$$
\begin{aligned}
\frac{\lambda}{p} \int_{\partial \Omega} e^{U_{\lambda}^{p}}= & \frac{\lambda}{p} \int_{\partial \Omega} e^{\gamma^{p}\left(1+\frac{1}{p \gamma^{p}} V_{\lambda}\left(\frac{x}{\varepsilon}\right)\right)^{p}} \\
= & \frac{\lambda}{p} \sum_{j=1}^{k} \int_{\partial \Omega \cap B\left(\xi_{j}, \tilde{\delta}\right)} e^{\gamma^{p}\left(1+\frac{1}{p \gamma^{p}} V_{\lambda}\left(\frac{x}{\varepsilon}\right)\right)^{p}} \\
& +\frac{\lambda}{p} \int_{\partial \Omega \backslash \bigcup_{j=1}^{k} B\left(\xi_{j}, \tilde{\delta}\right)} e^{\gamma^{p}\left(1+\frac{1}{p \gamma^{p}} V_{\lambda}\left(\frac{x}{\varepsilon}\right)\right)^{p}} \\
:= & I_{1}+I_{2} .
\end{aligned}
$$

First we observe that

$$
I_{2}=\lambda \Theta_{\lambda}(\xi)
$$

with $\Theta_{\lambda}(\xi)$ a function uniformly bounded as $\lambda \rightarrow 0$. On the other hand,

$$
\begin{aligned}
I_{1}= & \frac{1}{p^{2} \gamma^{2(p-1)}} \sum_{j=1}^{k} \int_{\partial \Omega_{\varepsilon} \cap B\left(\xi_{j}^{\prime}, \tilde{\delta} / \varepsilon\right)} e^{\gamma^{p}\left[\left(1+\frac{1}{p \gamma^{p}} V_{\lambda}(y)\right)^{p}-1\right]} \\
= & \frac{1}{p^{2} \gamma^{2(p-1)}} \sum_{j=1}^{k} \int_{\partial \Omega_{\varepsilon} \cap B\left(\xi_{j}^{\prime}, \tilde{\delta} / \varepsilon\right)} e^{\left\{\tilde{w}_{j}(y)+\frac{p-1}{p} \frac{1}{\gamma^{p}} \tilde{w}_{1 j}(y)+\left(\frac{p-1}{p}\right)^{2} \frac{1}{\gamma^{2 p}} \tilde{w}_{2 j}(y)+\theta(y)\right\}} \\
& \times\left(1+O\left(\frac{1}{\gamma^{p}}\right)\right) \\
= & \frac{1}{p^{2} \gamma^{2(p-1)}} \sum_{j=1}^{k} \int_{\partial \Omega_{\varepsilon} \cap B\left(0, \frac{\tilde{\delta}}{\mu_{j} \varepsilon}\right)} \frac{2}{|y-v(0)|^{2}}\left(1+O\left(\frac{1}{\gamma^{p}}\right)\right) \\
= & \frac{1}{p^{2} \gamma^{2(p-1)}} 2 k \pi\left(1+|\log \varepsilon|^{-1} \Theta_{\lambda}(\xi)\right),
\end{aligned}
$$

with $\Theta_{\lambda}(\xi)$ a function uniformly bounded as $\lambda \rightarrow 0$. From (5.19)-(5.21) we get

$$
\frac{\lambda}{p} \int_{\partial \Omega} e^{U_{\lambda}^{p}}=\frac{1}{p^{2} \gamma^{2(p-1)}}\left[2 k \pi\left(1+|\log \varepsilon|^{-1} \Theta_{\lambda}(\xi)\right)\right] .
$$


By (1.8), (5.8), (5.9), (5.18) and (5.22), we can write the whole asymptotic expansion of $F_{\lambda}(\xi)$, namely (5.6) holds.

\section{Proof of the main theorem}

Proof of Theorem 1.1. From Lemma 5.1, the function

$$
U_{\lambda}(\xi)+\tilde{\phi}(\xi)=\frac{1}{p \gamma^{p-1}}\left(p \gamma^{p}+\left(V_{\lambda}+\phi\right)\left(\frac{x}{\varepsilon}\right)\right)
$$

where $V_{\lambda}$ defined by (2.23) and $\phi(\xi)$ is the unique solution of problem (4.1), is a solution of problem (1.1) if we adjust $\xi$ so that it is a critical point of $F_{\lambda}(\xi)$ defined by (5.3). This is equivalent to finding a critical point of

$$
\widetilde{F}_{\lambda}(\xi):=A \lambda^{-1} \varepsilon^{\frac{2-p}{p}} F_{\lambda}(\xi)+B+C \log \varepsilon,
$$

for suitable constants $A, B$ and $C$. On the other hand, from Lemmas 5.2, for $\xi=$ $\left(\xi_{1}, \ldots, \xi_{k}\right) \in \partial \Omega$ satisfying $(2.2)$, we have that,

$$
\widetilde{F}_{\lambda}(\xi)=\varphi_{k}(\xi)+O\left(|\log \varepsilon|^{-1}\right) \Theta_{\lambda}(\xi),
$$

where $\varphi_{k}$ is given by (1.4), and $\Theta_{\lambda}(\xi)$ is uniformly bounded in the considered region as $\lambda \rightarrow 0$.

Next we show that $\varphi_{k}$ has at least two critical points. Assume $\xi_{1}, \ldots, \xi_{k}$ belong to the same component of $\partial \Omega$. We denote by $\Gamma$ such a component. Let $D$ be the diagonal in $(\partial \Omega)^{k}$. Define $\hat{\Omega}_{k}=(\partial \Omega)^{k} \backslash D$ and

$$
\tilde{\Omega}_{k}=\left\{\xi \in C^{k} \backslash D:\left|\xi_{i}-\xi_{j}\right|>\delta\right\}
$$

for some small but fixed $\delta>0$. We show that $\varphi_{k}$ has at least two critical points in $\tilde{\Omega}_{k}$.

First observe that $\varphi_{k}$ has an absolute maximum $m$ in $\tilde{\Omega}_{k}$ : indeed, $\varphi_{k}$ is $C^{1}$, it is bounded from above in $\hat{\Omega}_{k}$ (and hence in $\tilde{\Omega}_{k}$ ) and

$$
\varphi_{k}\left(\xi_{1}, \ldots, \xi_{k}\right) \rightarrow-\infty, \quad \text { as } \quad\left|\xi_{i}-\xi_{j}\right| \rightarrow 0 \text { for some } i \neq j .
$$

On the other hand, in our setting the number of critical points of $\varphi_{k}$ in $\tilde{\Omega}_{k}$ can be estimated from below by the Ljusternik-Schnirelman category of $\tilde{\Omega}_{k}$, namely the minimal number of closed and contractible sets in $\tilde{\Omega}_{k}$ whose union covers $\tilde{\Omega}_{k}$. In [6], it is proved that the Ljusternik-Schnirelman category of $\tilde{\Omega}_{k}$, cat $\left(\tilde{\Omega}_{k}\right)$, is greater than or equal to two, for any $k \geq 1$. Define

$$
c=\sup \Gamma \in \Xi \inf _{\xi \in \Gamma} \varphi_{k}(\xi)
$$


where

$$
\Xi=\left\{\Gamma \subset \tilde{\Omega}_{k}: \Gamma \quad \text { closed and } \quad \operatorname{cat}(\Gamma) \geq 2\right\} .
$$

Then $c$ is a critical level. If $c \neq m$, then $\varphi_{k}$ has at least two distinct critical points in ' $\tilde{\Omega}_{k}$. If $c=m$, then (6.2) gives the existence of a set $\Gamma$ with cat $(\Gamma) \geq 2$ where $\varphi_{k}$ reaches its maximum. In this case we conclude that there are infinitely many critical points for $\varphi_{k}$ in $\tilde{\Omega}_{k}$.

These critical points persist under small $C^{0}$-perturbation of the function, thus (6.1) gives that also the function $\widetilde{F}_{\lambda}(\xi)$ has two distinct critical points in $\tilde{\Omega}_{k}$, and hence problem (1.1) has at least two distinct solutions.

Expansion (1.9) follows from (1.8) and (5.22), while (1.10) holds as a direct consequence of the construction of $U_{\lambda}$. Expansion (1.11) is a consequence of (5.6).

Remark 6.1. Let us mention that one can get a stronger existence result assuming that the function $\varphi_{k}$ has some other critical points in $\tilde{\Omega}_{k}$, provided it is a topologically non trivial critical point for $\varphi_{k}$. We refer to [6] for further reference.

\section{Appendix}

Proof of (2.14). Since $(\arctan t)^{\prime}=\frac{1}{1+t^{2}}$, integration by parts gives

$$
\begin{aligned}
& \int_{0}^{\infty} \frac{1}{t^{2}+1} \log \frac{1}{t^{2}+1} d t=\int_{0}^{\infty} \log \frac{1}{t^{2}+1} d(\arctan t) \\
& =\left.\left[\arctan t \log \frac{1}{1+t^{2}}\right]\right|_{0} ^{+\infty}+\int_{0}^{+\infty} \frac{2 t \arctan t}{1+t^{2}} d t
\end{aligned}
$$

Setting $t=\tan x$ we have $1+t^{2}=\sec ^{2} t, d t=\sec ^{2} t d x$, hence

$$
\begin{aligned}
& \int_{0}^{+\infty} \frac{2 t \arctan t}{1+t^{2}} d t=2 \int_{0}^{\frac{\pi}{2}} x \tan x d x \\
& =-2 \int_{0}^{\frac{\pi}{2}} x d(\log (\cos x)) \\
& =\left.[-2 x \log (\cos x)]\right|_{0} ^{\frac{\pi}{2}}+2 \int_{0}^{\frac{\pi}{2}} \log (\cos x) d x \\
& =\left.[-2 \arctan t \log [\cos (\arctan t)]]\right|_{0} ^{+\infty}+2 \int_{0}^{\frac{\pi}{2}} \log (\cos x) d x \\
& =\left.\left[-\arctan t \log \left[\cos ^{2}(\arctan t)\right]\right]\right|_{0} ^{+\infty}+2 \int_{0}^{\frac{\pi}{2}} \log (\cos x) d x .
\end{aligned}
$$

We have

$$
\cos ^{2}(\arctan t)=\cos ^{2} x=\frac{1}{\sec ^{2} x}=\frac{1}{1+\tan ^{2} x}=\frac{1}{1+t^{2}} .
$$


On the other hand, we note that

$$
\int_{0}^{\frac{\pi}{2}} \log (\cos x) d x=\int_{0}^{\frac{\pi}{2}} \log (\sin x) d x .
$$

Then we have

$$
\begin{aligned}
& 2 \int_{0}^{\frac{\pi}{2}} \log (\cos x) d x \\
& =\int_{0}^{\frac{\pi}{2}} \log (\cos x) d x+\int_{0}^{\frac{\pi}{2}} \log (\sin x) d x \\
& =\int_{0}^{\frac{\pi}{2}} \log \left(\frac{\sin (2 x)}{2}\right) d x=\frac{1}{2} \int_{0}^{\pi} \log \left(\frac{\sin (x)}{2}\right) d x \\
& =\frac{1}{2} \int_{0}^{\pi} \log (\sin x) d x-\frac{\pi}{2} \log 2 \\
& =\int_{0}^{\frac{\pi}{2}} \log (\sin x) d x-\frac{\pi}{2} \log 2 \\
& =\int_{0}^{\frac{\pi}{2}} \log (\cos x) d x-\frac{\pi}{2} \log 2 .
\end{aligned}
$$

Hence we get

$$
\begin{aligned}
& \int_{-\infty}^{\infty} \frac{1}{t^{2}+1} \log \frac{1}{t^{2}+1} d t=2 \int_{0}^{\infty} \frac{1}{t^{2}+1} \log \frac{1}{t^{2}+1} d t \\
& =4 \int_{0}^{\frac{\pi}{2}} \log (\cos x) d x=-2 \pi \log 2 .
\end{aligned}
$$

Proof of Lemma 2.1. On the boundary, we have

$$
\frac{\partial H_{j}^{\varepsilon}}{\partial v}=\varepsilon e^{u_{j}}-\frac{\partial u_{j}}{\partial v}=2 \varepsilon \mu_{j} \frac{1-v\left(\xi_{j}\right) \cdot v(x)}{\left|x-\xi_{j}-\varepsilon \mu_{j} v\left(\xi_{j}\right)\right|^{2}}+2 \frac{\left(x-\xi_{j}\right) \cdot v(x)}{\left|x-\xi_{j}-\varepsilon \mu_{j} v\left(\xi_{j}\right)\right|^{2}} .
$$

Thus,

$$
\lim _{\varepsilon \rightarrow 0} \frac{\partial H_{j}^{\varepsilon}}{\partial \nu}=2 \frac{\left(x-\xi_{j}\right) \cdot v(x)}{\left|x-\xi_{j}\right|^{2}}, \quad \forall x \neq \xi_{j} .
$$

Set $z_{\varepsilon}(x)=H_{j}^{\varepsilon}(x)+\log \left(2 \mu_{j}\right)-H\left(x, \xi_{j}\right)$; then from the definition of $H\left(x, \xi_{j}\right)$ and $H_{j}^{\varepsilon}$ we have

$$
\begin{cases}-\Delta z_{\varepsilon}+z_{\varepsilon}=\log \frac{1}{\left|x-\xi_{j}\right|^{2}}-\log \frac{1}{\left|x-\xi_{j}-\varepsilon \mu_{j} \nu\left(\xi_{j}\right)\right|^{2}} & \text { in } \Omega \\ \frac{\partial z_{\varepsilon}}{\partial \nu}=\frac{\partial H_{j}^{\varepsilon}}{\partial \nu}-2 \frac{\left(x-\xi_{j}\right) \cdot v(x)}{\left|x-\xi_{j}\right|^{2}} & \text { on } \partial \Omega .\end{cases}
$$


First, we claim that there is a positive constant $C$ such that

$$
\left\|\frac{\partial H_{j}^{\varepsilon}}{\partial \nu}-2 \frac{\left(x-\xi_{j}\right) \cdot v(x)}{\left|x-\xi_{j}\right|^{2}}\right\|_{L^{q}(\partial \Omega)} \leq C \varepsilon^{1 / q}, \quad \forall q>1 .
$$

In fact,

$$
\begin{aligned}
& \frac{\partial H_{j}^{\varepsilon}}{\partial v}-2 \frac{\left(x-\xi_{j}\right) \cdot v(x)}{\left|x-\xi_{j}\right|^{2}} \\
& =2 \varepsilon \mu_{j} \frac{1-v\left(\xi_{j}\right) \cdot v(x)}{\left|x-\xi_{j}-\varepsilon \mu_{j} v\left(\xi_{j}\right)\right|^{2}}+2 \frac{\left(x-\xi_{j}\right) \cdot v(x)}{\left|x-\xi_{j}-\varepsilon \mu_{j} v\left(\xi_{j}\right)\right|^{2}}-2 \frac{\left(x-\xi_{j}\right) \cdot v(x)}{\left|x-\xi_{j}\right|^{2}} \\
& =2 \varepsilon \mu_{j} \frac{1-v\left(\xi_{j}\right) \cdot v(x)}{\left|x-\xi_{j}-\varepsilon \mu_{j} v\left(\xi_{j}\right)\right|^{2}}+2 \varepsilon \mu_{j} \frac{\left(x-\xi_{j}\right) \cdot v(x)\left[2\left(x-\xi_{j}\right) \cdot v(x)-\varepsilon \mu_{j}\right]}{\left|x-\xi_{j}\right|^{2}\left|x-\xi_{j}-\varepsilon \mu_{j} v\left(\xi_{j}\right)\right|^{2}} .
\end{aligned}
$$

Now, we observe that

$$
\left|1-v\left(\xi_{j}\right) \cdot v(x)\right| \leq C\left|x-\xi_{j}\right|^{2}, \quad\left|\left(x-\xi_{j}\right) \cdot v(x)\right| \leq C\left|x-\xi_{j}\right|^{2}, \quad \forall x \in \partial \Omega .
$$

Hence,

$$
\left|\frac{\partial H_{j}^{\varepsilon}}{\partial \nu}-2 \frac{\left(x-\xi_{j}\right) \cdot v(x)}{\left|x-\xi_{j}\right|^{2}}\right| \leq C \varepsilon+C \frac{\varepsilon\left|2\left(x-\xi_{j}\right) \cdot v\left(\xi_{j}\right)-\varepsilon \mu_{j}\right|}{\left|x-\xi_{j}-\varepsilon \mu_{j} \nu\left(\xi_{j}\right)\right|^{2}} .
$$

For small $\rho>0$, we have

$$
\left|\frac{\partial H_{j}^{\varepsilon}}{\partial v}-2 \frac{\left(x-\xi_{j}\right) \cdot v(x)}{\left|x-\xi_{j}\right|^{2}}\right| \leq C \varepsilon, \quad \text { for } \forall\left|x-\xi_{j}\right| \geq \rho, x \in \partial \Omega .
$$

Now take $q>1$; we have

$$
\begin{aligned}
& \int_{B_{\rho}\left(\xi_{j}\right) \cap \partial \Omega}\left|\frac{\varepsilon\left|2\left(x-\xi_{j}\right) \cdot v\left(\xi_{j}\right)-\varepsilon \mu_{j}\right|}{\left|x-\xi_{j}-\varepsilon \mu_{j} \nu\left(\xi_{j}\right)\right|^{2}}\right|^{q} d x \\
& =C \varepsilon \int_{B_{\rho / \varepsilon}(0) \cap \partial \Omega_{\varepsilon}}\left|\frac{2 y \cdot v(0)-\mu_{j}}{y-\mu_{j} \nu(0)}\right|^{q} d y \\
& \leq C \varepsilon \int_{0}^{\rho / \varepsilon} \frac{1}{(1+s)^{q}} d s \leq C \varepsilon .
\end{aligned}
$$

Combining (7.2) with (7.3) and (7.4) we conclude that (7.1) holds.

Next, we show that

$$
\begin{array}{r}
\left\|\log \frac{1}{\left|x-\xi_{j}\right|^{2}}-\log \frac{1}{\left|x-\xi_{j}-\varepsilon \mu_{j} \nu\left(\xi_{j}\right)\right|^{2}}\right\|_{L^{q}(\Omega)} \leq C \varepsilon \\
\text { for any } 1<q<2 .
\end{array}
$$


In fact, for $q \geq 1$, we write

$$
\begin{aligned}
& \left\|\log \frac{1}{\left|x-\xi_{j}\right|^{2}}-\log \frac{1}{\left|x-\xi_{j}-\varepsilon \mu_{j} v\left(\xi_{j}\right)\right|^{2}}\right\|_{L^{q}(\Omega)}^{q} \\
& =\int_{B_{10 \varepsilon \mu_{j}}\left(\xi_{j}\right) \cap \Omega} \ldots+\int_{\Omega \backslash B_{10 \varepsilon \mu_{j}}\left(\xi_{j}\right)} \ldots:=I+I I .
\end{aligned}
$$

Next we estimate $I$ and $I I$. For $I$, we observe that

$$
\int_{B_{10 \varepsilon \mu_{j}}\left(\xi_{j}\right) \cap \Omega}\left|\log \frac{1}{\left|x-\xi_{j}\right|^{2}}\right|^{q} d x \leq C \int_{0}^{C \varepsilon}|\log r|^{q} r d r \leq C \varepsilon^{2}\left(\log \frac{1}{\varepsilon}\right)^{q},
$$

and the same bound is true for the integral of $\left|\log \frac{1}{\left|x-\xi_{j}-\varepsilon \mu_{j} \nu\left(\xi_{j}\right)\right|^{2}}\right|^{q}$ in $B_{10 \varepsilon \mu_{j}}\left(\xi_{j}\right) \cap$ $\Omega$. Hence we have

$$
|I| \leq C \varepsilon^{2}\left(\log \frac{1}{\varepsilon}\right)^{q} .
$$

For $I I$, if $\left|x-\xi_{j}\right| \geq 10 \varepsilon \mu_{j}$, we have

$$
\left|x-\xi_{j}\right| \leq\left|x-\xi_{j}-\operatorname{t\varepsilon } \mu_{j} v\left(\xi_{j}\right)\right|+\mu_{j} \varepsilon \leq\left|x-\xi_{j}-t \varepsilon \mu_{j} v\left(\xi_{j}\right)\right|+\frac{1}{10}\left|x-\xi_{j}\right|
$$

for any $t \in[0,1]$, then we have $\left|x-\xi_{j}\right| \leq C\left|x-\xi_{j}-t \varepsilon \mu_{j} v\left(\xi_{j}\right)\right|$. Using this fact, we can obtain

$$
\begin{aligned}
& \left|\log \frac{1}{\left|x-\xi_{j}\right|^{2}}-\log \frac{1}{\left|x-\xi_{j}-\varepsilon \mu_{j} v\left(\xi_{j}\right)\right|^{2}}\right| \\
& \leq C \sup _{0 \leq t \leq 1} \frac{C \varepsilon}{\left|x-\xi_{j}-\varepsilon \mu_{j} v\left(\xi_{j}\right)\right|} \leq \frac{C \varepsilon}{\left|x-\xi_{j}\right|} .
\end{aligned}
$$

Thus for $1<q<2$,

$$
|I I| \leq C \varepsilon^{q} \int_{10 \varepsilon \mu_{j}}^{D} r^{1-q} d r \leq C \varepsilon^{q},
$$

where $D$ is the diameter of $\Omega$. Thus, combining (7.6) with (7.7) and (7.8) we obtain that (7.5) holds.

Therefore by elliptic regularity theory, we obtain

$$
\left\|z_{\varepsilon}\right\|_{W^{1+s, q(\Omega)}} \leq\left(\left\|\frac{\partial z_{\varepsilon}}{\partial v}\right\|_{L^{q}(\partial \Omega)}+\left\|\Delta z_{\varepsilon}\right\|_{L^{q}(\Omega)}\right) \leq C \varepsilon^{1 / q}
$$

for any $0<s<\frac{1}{q}$. By the Morrey embedding we obtain

$$
\left\|z_{\varepsilon}\right\|_{C^{\beta}}(\bar{\Omega}) \leq C \varepsilon^{1 / q}
$$

for any $0<\beta<\frac{1}{2}+\frac{1}{q}$. This proves the lemma with $\alpha=\frac{1}{q}$. 
Proof of Lemma 2.4. The proof follows from the same arguments as those used to prove Lemma 2.1. First, on the boundary, we have

$$
\lim _{\varepsilon \rightarrow 0} \frac{\partial H_{j}^{\varepsilon}}{\partial v}(x)=2 \alpha_{i j} \frac{\left(x-\xi_{j}\right) \cdot v(x)}{\left|x-\xi_{j}\right|^{2}}, \quad \forall x \neq \xi_{j} .
$$

The regular part of Green's function satisfies

$$
\begin{cases}-\Delta_{x} H\left(x, \xi_{j}\right)+H\left(x, \xi_{j}\right)=-\log \frac{1}{\left|x-\xi_{j}\right|^{2}} & \text { in } \Omega \\ \frac{\partial H\left(x, \xi_{j}\right)}{\partial v_{x}}=2 \frac{\left(x-\xi_{j}\right) \cdot v(x)}{\left|x-\xi_{j}\right|^{2}} & \text { on } \partial \Omega .\end{cases}
$$

Setting $\tilde{z}_{\varepsilon}=H_{j}^{\varepsilon}(x)+\alpha_{i j} \log \left(2 \mu_{j} \varepsilon^{2}\right)-\alpha_{i j} H\left(x, \xi_{j}\right)$, we have

$$
\begin{aligned}
-\Delta \tilde{z}_{\varepsilon}+\tilde{z}_{\varepsilon} & =-\Delta H_{j}^{\varepsilon}+H_{j}^{\varepsilon}+\alpha_{i j} \log \left(2 \mu_{j} \varepsilon^{2}\right)-\alpha_{i j}\left[-\Delta H\left(x, \xi_{j}\right)+H\left(x, \xi_{j}\right)\right] \\
& =-\alpha_{i j} \tilde{w}_{j}+\alpha_{i j} \log \left(2 \mu_{j} \varepsilon^{2}\right)-\alpha_{i j}\left[-\Delta H\left(x, \xi_{j}\right)+H\left(x, \xi_{j}\right)\right] \\
& =\alpha_{i j}\left[\log \frac{1}{\left|x-\xi_{j}\right|^{2}}-\log \frac{1}{\left|x-\xi_{j}-\varepsilon \mu_{j} \nu\left(\xi_{j}\right)\right|^{2}}\right] \quad \text { in } \Omega .
\end{aligned}
$$

On the other hand, on the boundary we have

$$
\frac{\partial \tilde{z}_{\varepsilon}}{\partial v}=\alpha_{i j}\left[\frac{\partial H_{j}^{\varepsilon}}{\partial v}-2 \frac{\left(x-\xi_{j}\right) \cdot v(x)}{\left|x-\xi_{j}\right|^{2}}\right] .
$$

From (7.1) and (7.9), by the same procedure as in the proof of Lemma 2.1, we obtain that (2.20) holds.

\section{References}

[1] Adimurthi and S. L. Yadava, Critical exponent problem in $\mathbb{R}^{2}$ with Neumann boundary condition, Comm. Partial Differential Equations 15 (1990), 461-501.

[2] A. B AHRI and J. M. CORON, On a nonlinear elliptic equation involving the critical Sobolev exponent: The effect of the topology of the domain, Comm. Pure Appl. Math. 41 (1988), 255-294.

[3] S. BARAKET and F. PACARD, Construction of singular limits for a semilinear elliptic equation in dimension 2, Calc. Var. Partial Differential Equations 6 (1998), 1-38.

[4] H. BREZIS and F. MERLE, Uniform estimates and blow-up behavior for solutions of $-\Delta u=V(x) e^{u}$ in two dimensions, Comm. Partial Differential Equations 16 (1991), 12231253.

[5] H. CASTRO, Solutions with spikes at the boundary for a $2 D$ nonlinear Neumann problem with large exponent, J. Differential Equations 246 (2009), 2991-3037.

[6] J. DÁvila, M. DEL PINo and M. Musso, Concentrating solutions in a two-dimensional elliptic problem with exponential Neumann data, J. Funct. Anal. 227 (2005), 430-490. 
[7] J. DÁvila, M. Del Pino, M. Musso and J. WeI, Singular limits of a two-dimensional boundary value problem arising in corrosion modelling, Arch. Ration. Mech. Anal. 182 (2006), 181-221.

[8] M. DEL Pino, M. KowalCZYK and M. Musso, Singular limits in Liouville-type equations, Calc. Var. Partial Differential Equations 24 (2005), 47-81.

[9] M. DEL PINo, M. Musso and B. RuF, New solutions for Trudinger-Moser critical equations in $\mathbb{R}^{2}$, J. Funct. Anal. 258 (2010), 421-457.

[10] S. DENG and M. Musso, Bubbling solutions for an exponential nonlinearity in $\mathbb{R}^{2}, \mathrm{~J}$. Differential Equations (2014), DOI: 10.1016/j.jde.2014.05.034

[11] P. Esposito, M. Grossi and A. Pistoia, On the existence of blowing-up solutions for a mean field equation, Ann. Inst. H. Poincaré Anal. Non Linéaire 22 (2005), 227-257.

[12] P. Esposito, M. Musso and A. Pistoia, Concentrating solutions for a planar elliptic problem involving nonlinearities with large exponent, J. Differential Equations 227 (2006), $29-68$.

[13] P. Esposito, M. Musso and A. Pistoia, On the existence and profile of nodal solutions for a two-dimensional elliptic problem with large exponent in nonlinearity, Proc. Lond. Math. Soc. 94 (2007), 497-519.

[14] P. Esposito, A. PistoiA and J. WeI, Concentrating solutions for the Hénon equation in $\mathbb{R}^{2}$, J. Anal. Math. 100 (2006), 249-280.

[15] A. FLOER and A. WeINSTEIN, Nonspreading wave packets for the cubic Schrödinger equation with a bounded potential, J. Funct. Anal. 69 (1986), 397-408.

[16] Y. Y. LI and I. SHAFRIR, Blow-up analysis for solutions of $-\Delta u=V e^{u}$ in dimension two, Indiana Univ. Math. J. 43 (1994), 1255-1270.

[17] M. MUSSO and J. WEI, Stationary solutions to a Keller-Segel chemotaxis system, Asymptot. Anal. 49 (2006), 217-247.

[18] K. NAGASAKI and T. SUZUKI, Asymptotic analysis for two-dimensional elliptic eigenvalue problems with exponentially dominated nonlinearities, Asymptot. Anal. 3 (1990), 173-188.

Departamento de Ingeniería Matemática and CMM

Universidad de Chile

Casilla 170 Correo 3

Santiago, Chile

shbdeng@yahoo.com.cn

Departamento de Matemática

Pontificia Universidad Catolica de Chile

Avda. Vicuña Mackenna

4860, Macul, Chile

mmusso@mat.puc.cl 